\title{
First-line chemotherapy regimens for locally advanced and metastatic pancreatic adenocarcinoma: a Bayesian analysis
}

This article was published in the following Dove Press journal:

Cancer Management and Research

\begin{abstract}
Shuisheng Zhang, ${ }^{1,2}$ Weimin Xie, ${ }^{3}$ Yinghua Zou, ${ }^{4}$ Shuanghua Xie, ${ }^{5}$ Jianwei Zhang,' Wei Yuan, ${ }^{6,7}$ Jie $M a,{ }^{6-8}$ Jiuda Zhao, ${ }^{9}$ Cuiling Zheng, ${ }^{10}$ Yingtai Chen,' Chengfeng Wang'

'Department of Pancreatic and Gastric Surgery, National Cancer Center/Cancer Hospital, Chinese Academy of Medical

Sciences and Peking Union Medical College, ${ }^{2}$ Department of General Surgery, Peking University Third Hospital, ${ }^{3}$ Department of Obstetrics and Gynecology, Peking Union Medical College Hospital, ${ }^{4}$ Department of Interventional Radiology and Vascular Surgery, Peking University First Hospital, ${ }^{5}$ Department of Cancer Epidemiology and Health Statistics, ${ }^{6}$ State Key Laboratory of Molecular Oncology, National Cancer Center/Cancer Hospital, Chinese Academy of Medical Sciences and Peking Union Medical College, ${ }^{7}$ Clinical Immunology Center, Chinese Academy of Medical Science, ${ }^{8}$ Department of Biotherapy, Beijing Hospital, National Center of Gerontology, Beijing, 'Department of Medical Oncology, Affiliated Hospital of Qinghai University, Xining, ${ }^{10}$ Department of Clinical Laboratory, National Cancer Center/Cancer Hospital, Chinese Academy of Medical Sciences and Peking Union Medical College, Beijing, China
\end{abstract}

Correspondence: Chengfeng Wang; Yingtai Chen

Department of Pancreatic and Gastric Surgery, National Cancer Center/Cancer Hospital, Chinese Academy of Medical Sciences and Peking Union Medical College, I7 Panjiayuan Nanli, Chaoyang District, Beijing I0002I, China

Email lifeofwater@I26.com; yzchenyingtai@I26.com
Background: Systemic chemotherapy is the standard treatment for locally advanced and metastatic pancreatic cancer, but there is no consensus on the optimum regimen. We aimed to compare and rank the locally advanced and metastatic pancreatic adenocarcinoma chemotherapy regimens evaluated in randomized controlled trials (RCTs) in the past 15 years.

Materials and methods: PubMed, Embase, Cochrane Collaboration database, and ClinicalTrials.gov were searched for RCTs comparing chemotherapy regimens as first-line treatment for locally advanced and metastatic pancreatic adenocarcinomas. By using Bayesian network meta-analysis, we compared and ranked all included chemotherapy regimens in terms of overall survival, progression-free survival, response rate, and hematological toxicity.

Results: The analysis included $68 \mathrm{RCTs}$, with 14,908 patients and 63 treatment strategies. For overall survival, NSC-631570 (hazard ratio [HR] vs gemcitabine monotherapy $0.44,95 \%$ credible interval: $0.24-0.76$ ) and gemcitabine+NSC-631570 (HR 0.45, 0.24-0.86) were the two top-ranked chemotherapy regimens. For progression-free survival, PEFG (cisplatin + epirubicin + fluorouracil + gemcitabine) ranked first (HR 0.51, 0.34-0.77). PG (gemcitabine + pemetrexed) (odds ratio [OR] 4.68, 2.24-9.64) and FLEC (fluorouracil + leucovorin + epirubicin + carboplatin) (OR 4.52, 1.14-24.00) were ranked the most hematologically toxic, with gastrazole having the least toxicity (OR 0.03, 0.00-0.46).

Conclusion: The chemotherapy regimens NSC-631570 and gemcitabine+NSC-631570 were ranked the most efficacious for locally advanced and metastatic pancreatic adenocarcinomas in terms of overall survival, which warrants further confirmation in large-scale RCTs.

Keywords: locally advanced and metastatic pancreatic adenocarcinoma, chemotherapy regimen, overall survival, rank

\section{Introduction}

\section{Rationale}

Pancreatic adenocarcinomas are mostly at the advanced stage when diagnosed. The prognosis is poor, with a 5-year survival rate of around $8 \% .{ }^{1}$ Although systemic chemotherapy is the most important first-line treatment for locally advanced and metastatic pancreatic adenocarcinomas, there is no consensus about which chemotherapy regimen is the most effective.

Randomized controlled trials (RCTs) have compared various chemotherapy regimens, providing important direct evidence. However, the number of RCTs is limited and direct comparisons between many of the regimens are lacking.

Meta-analysis provides a method for summarizing available evidence as a supplement for individual RCTs. However, traditional pairwise meta-analysis is limited in 
value because it only compares two groups of treatments, and a lack of direct one-to-one comparison studies can make certain comparisons of available treatments impossible. Thus, the traditional meta-analysis method may not be suitable for analyzing and summarizing the efficacy of the several chemotherapy regimens simultaneously for pancreatic adenocarcinomas.

Network meta-analysis offers a solution to these problems, allowing direct and indirect comparisons to be combined, so that several treatments can be compared simultaneously. ${ }^{2-4}$ When there is no available direct comparison between two therapies, network meta-analysis allows an indirect comparison. Bayesian analysis can be applied to network meta-analysis, allowing for inclusion of trials with more than two treatment arms, multiple comparisons, and more precise and stable outcomes. More importantly, Bayesian network meta-analysis can combine different measures of survival (hazard ratio [HR] and median survival duration) in a single analysis, avoiding the need for separate analyses for studies with different measurement methods and avoiding potential selection bias from only including studies with the same measurement methods. ${ }^{5}$

There have been two previous network meta-analyses of systemic regimens for locally advanced and metastatic pancreatic cancer. The first only included 22 studies and compared nine regimens, and it did not compare response rates. ${ }^{6} \mathrm{~A}$ recent network meta-analysis by Liu et al compared 12 chemotherapy regimens from 20 included studies. ${ }^{7}$ The previous meta-analyses synthesized the data provided by trials comparing chemotherapy regimens, but did not provide a data summary or a ranking of the efficacy of chemotherapy regimens for locally advanced and metastatic pancreatic cancer covering all available studies published over a long interval and including multiple outcomes.

\section{Objectives}

The aim of the present study, therefore, was to use a network meta-analysis to compare as comprehensively as possible the available systemic chemotherapy regimens for locally advanced and metastatic pancreatic adenocarcinoma, summarizing and ranking them in terms of overall survival, progression-free survival, response rate, and grade 3-4 hematological toxic effects, to provide some objective clues or references for future research and clinical practice. We included all the studies we could find that were published in the last 15 years; this resulted in an analysis of 63 regimens, the largest number analyzed to date.

\section{Materials and methods}

\section{Study design}

The analysis was designed to rank chemotherapy regimens by overall survival, progression-free survival, response rate, and grade 3-4 hematological toxicities using Bayesian network meta-analysis.

\section{Search strategy and selection criteria}

This study was conducted in accordance with the Preferred Reporting Items for Systematic reviews and Meta-Analyses guidelines. ${ }^{8}$ We searched the PubMed, Embase, Cochrane Collaboration database, ClinicalTrials.gov, and the reference lists of all relevant articles, including relevant reviews, for RCTs that compared systemic chemotherapy regimens for advanced pancreatic adenocarcinoma that included both locally advanced pancreatic adenocarcinoma and metastatic pancreatic adenocarcinoma as first treatments (or compared regimens with placebo). The search was for articles published in English between January 1, 2002, and May 2, 2017. The reasons for including RCTs published in last 15 years, the search terms, a description of the selection process, and a summary of results are shown in Supplementary S1. Relevant reports published on the US Food and Drug Administration website were searched for additional publications.

We included RCTs that compared two or more systemic chemotherapy regimens as first-line treatments for locally advanced and metastatic pancreatic adenocarcinomas, or that compared a regimen or regimens with placebo. To increase the number of trials and treatment methods that could be analyzed within the network, we also included a typical randomized clinical trial published in 1995 that compared gemcitabine and 5-fluorouracil (5-FU). This connected gemcitabine and 5-FU; otherwise, two networks would have been required. We excluded RCTs investigating metastatic pancreatic cancer only. We also excluded studies if they considered only one systemic regimen, or compared second-line treatments, or did not use randomization for allocating the treatment.

\section{Data extraction, outcomes, and assessment for risk of bias}

Two investigators selected the articles, reviewed the full reports of the included studies, and independently collected information into electronic spreadsheets. Disagreements such as the basic trial information or the HR or odds ratio (OR) to be extracted from a trial were resolved by discussion between the two investigators. The process if a consensus could not 
be reached was for a third investigator to help resolve the discrepancy, with the authority to make the final decision. Supplementary S2 lists the data extracted from the articles. For reports of the same RCT published at different times, we gave precedence to the updated data.

The primary outcomes of the meta-analysis were overall survival and progression-free survival after the systemic treatment of locally advanced and metastatic pancreatic adenocarcinoma. For this, our preferred outcome measure was the reported HRs, because HRs provide time-to-event information and take censoring into account. ${ }^{5}$ When an article did not report HRs, we estimated them from the survival curves using the methods of Tierney et al. ${ }^{9}$ If neither HRs nor survival curves were reported, we collected information about median survival durations. The secondary outcomes were response rate and hematological toxicity; the extraction methods for these are presented in Supplementary S2.

Two investigators independently assessed the risk of bias for the included studies using the Cochrane Risk of Bias method. ${ }^{10}$ Disagreement was resolved by discussion between the two investigators or by the intervention of a third investigator to reach consensus.

\section{Data synthesis and analysis}

For the meta-analyses of overall survival and progression survival, we used both fixed-effects and random-effects models. A random-effects model was used because of potential heterogeneity. The results obtained with a fixed-effects model are shown in the supplement. By using the approach described by Woods et al, ${ }^{5}$ different measures (HRs and survival duration) could be combined into a single-network meta-analysis, avoiding potential selection bias. For the metaanalyses of response rate and toxic effects, we calculated and compared the ORs and 95\% credible interval (CrI) using the WinBUGS model described by Chaimani. ${ }^{11}$

We also performed a traditional pairwise random-effects meta-analysis of direct comparisons. The HRs and ORs were calculated respectively for all outcomes, each with a $95 \%$ $\mathrm{CI}$, and we evaluated the heterogeneity of each pairwise comparison. ${ }^{12} \mathrm{~A}$ comparison of the direct comparison results with the pooled HRs and OR from the network meta-analysis allowed a rough assessment of any inconsistency between the direct and indirect comparisons.

To ensure study comparability, the RCT selection criteria were strictly followed and heterogeneity was evaluated. To detect clinical heterogeneity, the baseline characteristics of the RCT subjects were compared and the $\mathrm{I}^{2}$ statistics were calculated. To account for other heterogeneities that are difficult to detect, a random-effects model was used to analyze primary outcomes in the main text. Results obtained with a fixed-effects model are shown in the supplement.

To assess the convergence of the Bayesian model, we evaluated the trace plots and the Brooks-Gelman-Rubin statistic. ${ }^{13}$ We evaluated each model by comparing the number of data points and the mean posterior deviance, and compared the deviance information criterion between the fixed-effects and random-effects models. We assessed the ranking probabilities for each chemotherapy regimen for each outcome, reporting the rank as the surface under the cumulative ranking curve. ${ }^{14}$ We plotted a funnel plot for each outcome to detect any publication bias in the network meta-analysis. ${ }^{14}$

The traditional pairwise meta-analysis was performed using Stata 13.1 (StataCorp LCC, College Station, TX, USA) with a meta fixed- and random-effects meta-analysis package (http://www.stata-journal.com/article.html?article=sbe24_2), and the Bayesian network meta-analysis was performed using WinBUGS version 1.4.3 (Imperial College and MRC, Cambridge, UK), supplemented with ITC 2.0 (Canadian Agency for Drugs and Technologies in Health, Ottawa, Canada). In the network meta-analysis, we used non-informative uniform and normal prior distributions. ${ }^{2,3}$ We compiled two Markov chains and generated two sets of initial values to fit the model. For the parameter setting, we used 5,000 burn-ins and a thinning interval of 6-25 for each chain, updating to 35,000-55,000 iterations. The detailed parameters for each outcome measure in the network meta-analysis are listed in Table S2.

\section{Results \\ Study selection and study characteristics}

The search of databases and other resources identified a total of 2,133 papers (Figure 1; Supplementary S1). Initial screening of the titles and abstracts resulted in the exclusion of 1,971 of these. The remaining 162 potentially eligible articles were subjected to a detailed assessment of the full text, resulting in the final inclusion of 68 publications, reporting 68 RCTs. These compared 63 systemic chemotherapy regimens or placebo (Figure 2; Tables 1 and 2; Supplementary S3). The risks of bias for the studies are shown in Supplementary S4. The most common high risk was related to the blinding of participants and personnel (performance bias).

\section{Participants}

The included studies involved a total of 14,909 patients randomized to receive one of the 63 systemic chemotherapy 


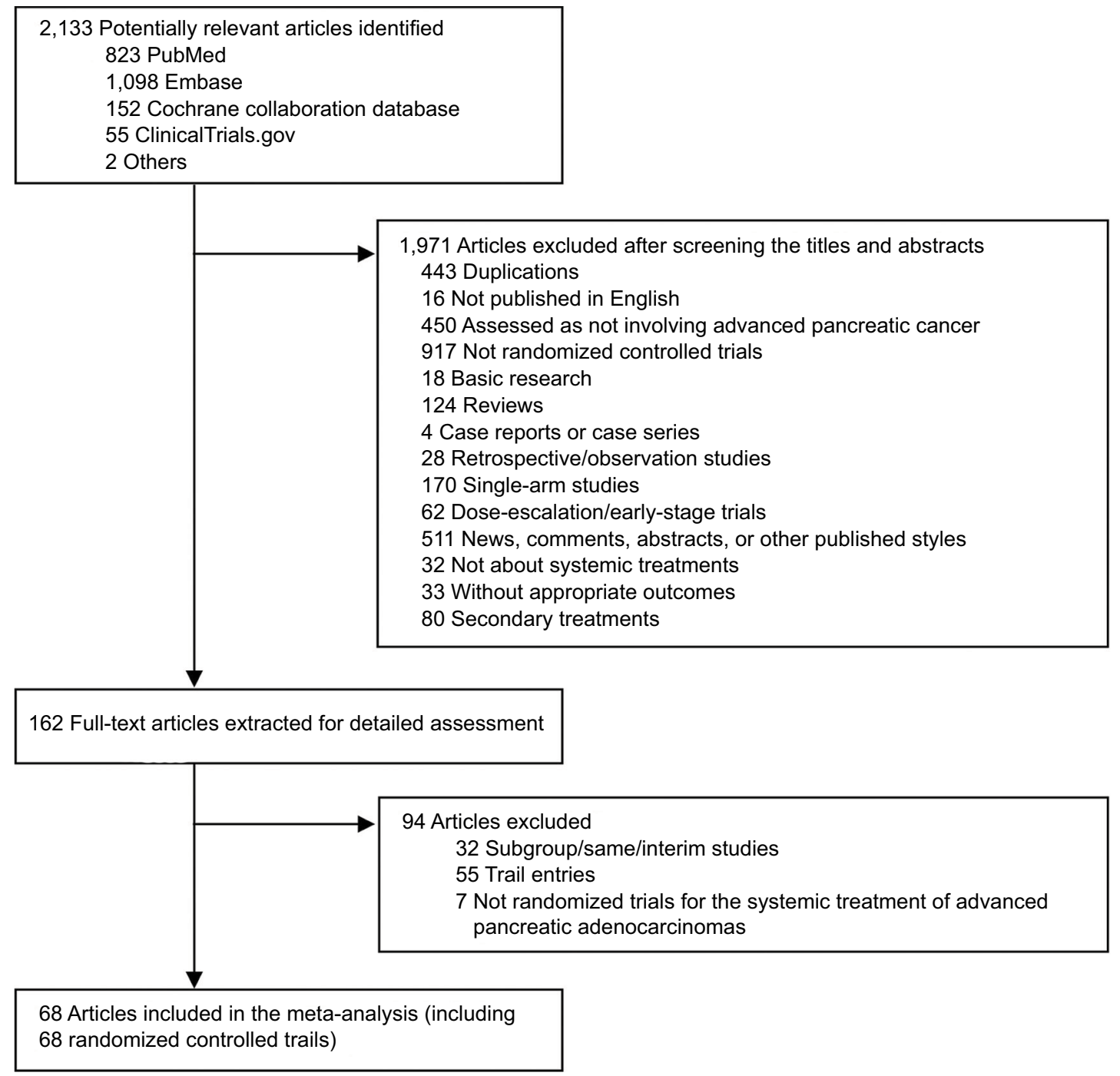

Figure I Literature search and selection.

regimens or placebo. The mean sample size was 219 participants (range 18-832), and $56.5 \%$ of the participants overall were male. The maximum and minimum median ages across all the included RCTs were 67.4 and 49.9 years, respectively. Around $72.8 \%$ of the total participants had metastatic pancreatic adenocarcinomas (Table 1).

\section{Primary outcomes}

Supplementary S3 lists the included studies. The network meta-analysis for overall survival included 62 studies comparing 54 treatments that reported overall survival information (Supplementary S5). In 61 of these studies, HRs for deaths could be found directly or calculated indirectly; the remaining study reported overall survival duration. Supplementary S6 presents the detailed results of the fixed- or random-effects network meta-analysis for overall survival. The 13 top-ranked chemotherapy regimens (by surface under the cumulative ranking curve) are shown in Figure 3, which also includes gemcitabine and the lowest-ranked regimen as references. Compared with gemcitabine, the following chemotherapy regimens had a high ranking because of increased overall survival: NSC-631570 (HR vs gemcitabine monotherapy $0.44,95 \%$ CrI 0.24-0.76), gemcitabine+NSC-631570 (HR $0.45,0.24-0.86$ ), PEFG (cisplatin + epirubicin + fluorouracil + gemcitabine; HR 0.63, 0.41-0.96), FLEC (fluorouracil + leucovorin + epirubicin + carboplatin; HR 0.65, 0.43-0.98), GemCape (gemcitabine + capecitabine; HR 0.82, 0.74-0.92), gemcitabine + erlotinib (HR 0.83, 0.69-0.99), and GS (gemcitabine+S-1, HR 0.84, 0.73-0.98), as shown in Figure 3 and Supplementary S6. Compared with gemcitabine, placebo was the lowest ranked chemotherapy regimen for improving overall survival (HR 7.14, 2.13-25.00). 


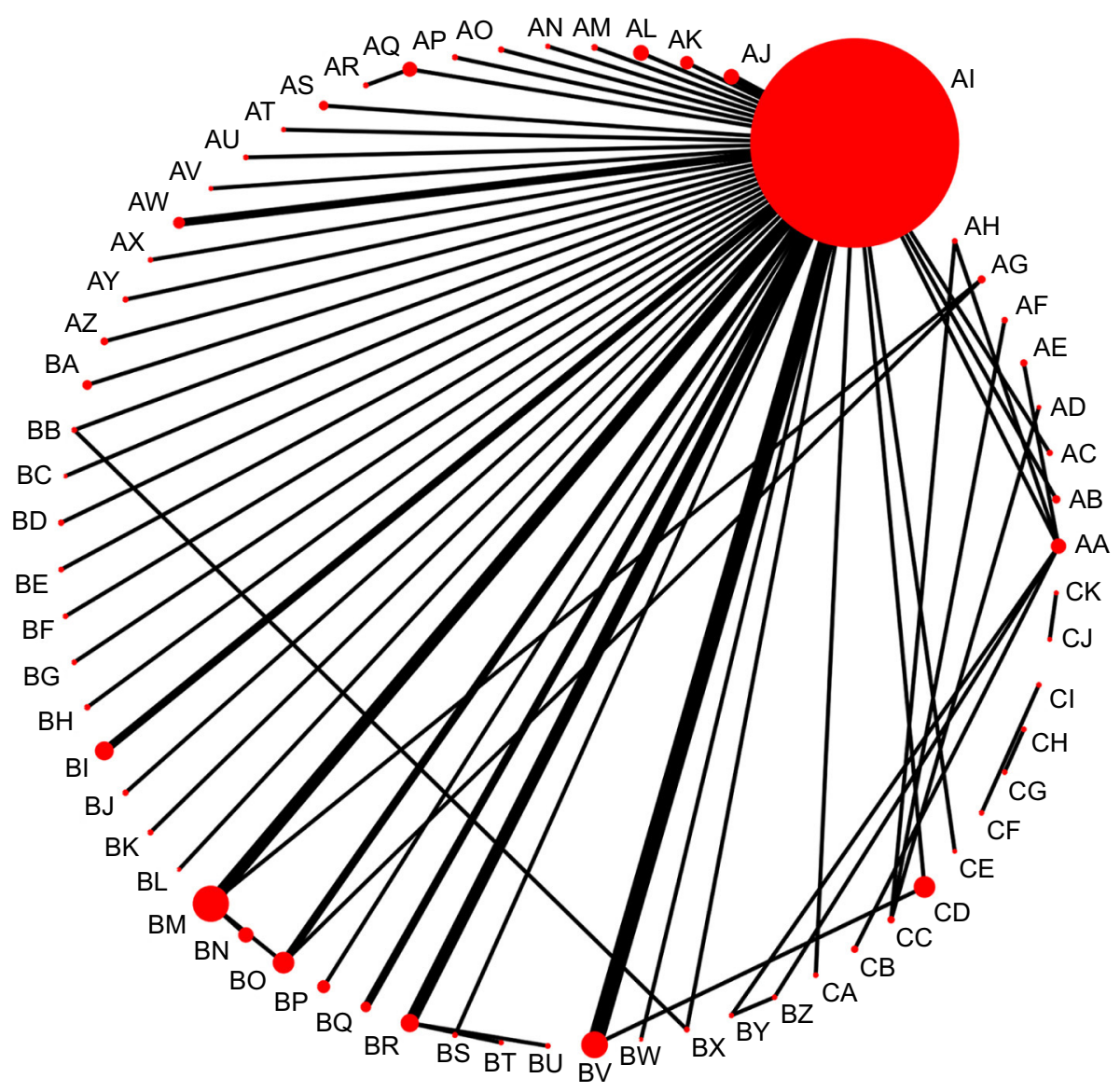

Figure 2 Network of comparisons for all the included studies.

Notes: The size of each circle corresponds to the sample size, with the width of the lines proportional to the number of trials containing related comparisons. An explanation of the two-letter codes is provided in Table 2.

The analysis of progression-free survival included 37 studies that compared 30 treatments strategies (Supplementary S5). Compared with gemcitabine, the following chemotherapy regimens had the highest ranking, with longer progression-free survival: PEFG (HR 0.51, 0.34-0.77), gemcitabine+TH-302 (HR 0.59, 0.40-0.87), GS (HR 0.63, 0.55-0.74), gemcitabine+IMM-101 (HR $0.58,0.38-0.92$ ), gemcitabine+5-FU (HR 0.71, 0.57-0.88), GemCape (HR 0.77, 0.63-0.92), and GemOX (gemcitabine + oxaliplatin, HR 0.86, 0.75-0.97), as shown in Figure 3 and Supplementary S7. BAY 12-9566 had the lowest efficacy ranking, with the shortest progression-free survival compared with gemcitabine (HR 1.89, 1.47-2.44).

\section{Secondary outcomes}

Information on the overall response rate was reported by 50 studies, which compared 42 chemotherapy regimens; disease control rate was reported in 44 studies comparing 43 treatment strategies (Supplementary S5). Compared with gemcitabine, the following chemotherapy regimens had the highest ranking, with increased overall response rates: PEFG (OR 7.16), gemcitabine+NSC-631570 (OR 10.36), GS (OR 3.49), gemcitabine + axitinib (OR 3.19), gemcitabine + irinotecan (OR 2.94), PG (gemcitabine + pemetrexed, OR 2.30), and GemCape (OR 2.15), as shown in Figure 4 and Supplementary S8. Similarly, the following regimens had the highest improved disease control rate ranking: NSC-631570 (OR 7.10), PEFG (OR 4.27), GS (OR 2.65), and GemCis (gemcitabine + cisplatin, OR 2.20), as shown in Figure 4 and Supplementary S8. BAY 12-9566 was the lowest ranked regimen for improving the overall response rate (OR 0.13) and disease control rate (HR 0.28) compared with gemcitabine.

The meta-analysis of hematological toxicity included 58 studies that reported information on grade 3-4 hematological toxicity, which compared 50 treatment strategies (Supplementary S5 and S9; Figure 5). Compared with gemcitabine, the following regimens were ranked the most likely to result in grade 3-4 hematological toxic effects: PG (OR 4.68), FLEC (OR 4.52), gemcitabine+TH-302 (OR 3.12), gemcitabine + exatecan (OR 2.58), GS (OR 2.40), 
Table I Randomized controlled trials included in the systematic review and network meta-analysis

\begin{tabular}{|c|c|c|c|c|c|}
\hline Study & $\begin{array}{l}\text { Mean or median } \\
\text { age }\end{array}$ & $\begin{array}{l}\text { Male/female } \\
\text { (ratio \%) }\end{array}$ & $\begin{array}{l}\text { Metastatic/advanced } \\
\text { (ratio \%) }\end{array}$ & $\begin{array}{l}\text { Total number } \\
\text { of participants }\end{array}$ & Comparison \\
\hline Burris et al $1997^{18}$ & 61.5 & 117 & 74 & 126 & $\mathrm{Al}$ vs $\mathrm{AA}$ \\
\hline Gansauge et al $2002^{19}$ & 60.9 & 58 & 62 & 90 & $A l$ vs $B X$ vs $B B$ \\
\hline Colucci $2002^{\prime}$ & 62.0 & 73 & 54 & 107 & Al vs BR \\
\hline Bramhall $2002^{2}$ & 62.0 & 71 & 61 & 239 & $\mathrm{AZ}$ vs $\mathrm{Al}$ \\
\hline Maisey $2002^{3}$ & 61.5 & 60 & 65 & 209 & $A A$ vs $A E$ \\
\hline Berlin $2002^{4}$ & 65.1 & 90 & 90 & 322 & $A l$ vs $B Q$ \\
\hline Ducreux $2002^{5}$ & 60.0 & 55 & 87 & 207 & $A A$ vs $C B$ \\
\hline Smith $2003^{6}$ & 60.3 & 62 & 76 & 55 & CE vs Al \\
\hline Moore $2003^{7}$ & 66.0 & 75 & 63 & 277 & $A B$ vs $A l$ \\
\hline Ducreux $2004^{8}$ & 57.0 & 50 & 91 & 63 & $B Y$ vs $B Z$ vs $A A$ \\
\hline Van Cutsem 20049 & 62.0 & 75 & 77 & 688 & $\mathrm{BI}$ vs $\mathrm{Al}$ \\
\hline Rocha Lima $2004^{10}$ & 61.7 & 73 & 81 & 360 & $A W$ vs $A l$ \\
\hline Cantore $2004^{\prime \prime}$ & 62.5 & 50 & 51 & 138 & Al vs $A C$ \\
\hline Chen $2006^{12}$ & 61.2 & 53 & 69 & 26 & $\mathrm{BW}$ vs $\mathrm{Al}$ \\
\hline Louvet $2005^{13}$ & 60.7 & 77 & 69 & 313 & $\mathrm{Al}$ vs $\mathrm{BO}$ \\
\hline Reni et al $2005^{20}$ & 60.6 & 106 & 71 & 99 & CA vs Al \\
\hline Di Costanzo $2005^{14}$ & 63.0 & 82 & 70 & 91 & $A l$ vs $B Q$ \\
\hline Oettle et al $2005^{35}$ & 63.0 & 76 & 91 & 565 & $\mathrm{BP}$ vs $\mathrm{Al}$ \\
\hline Negi et al $2006^{34}$ & 49.9 & 31 & 46 & 46 & $A D$ vs $C C$ \\
\hline Lutz et al $2006^{15}$ & 58.0 & 78 & 83 & 96 & $\mathrm{CF}$ vs $\mathrm{Cl}$ \\
\hline Chau 2006 Trial I ${ }^{15}$ & 62.0 & 39 & 33 & 18 & $\mathrm{AH}$ vs $\mathrm{CC}$ \\
\hline Chau 2006 Trial $2^{15}$ & 64.9 & 86 & 62 & 95 & $\mathrm{AH}$ vs $\mathrm{AA}$ \\
\hline Richards $2006^{16}$ & 63.5 & 67 & 83 & 174 & AM vs Al \\
\hline Stathopoulos $2006^{17}$ & 64.0 & 61 & 82 & 130 & $A W$ vs $\mathrm{Al}$ \\
\hline Heinemann $2006^{18}$ & 65.0 & 57 & 77 & 190 & $B R$ vs $A l$ \\
\hline Abou-Alfa $2006^{19}$ & 62.7 & 82 & 79 & 349 & AS vs Al \\
\hline Friess $2006^{20}$ & 67.0 & 102 & 80 & 89 & AN vs $\mathrm{Al}$ \\
\hline Moore et al $2007^{28}$ & 63.9 & 110 & 76 & 569 & $A Q$ vs $A I$ \\
\hline Herrmann et al $2007^{22}$ & NA & 87 & 65 & 319 & $\mathrm{BM}$ vs $\mathrm{Al}$ \\
\hline Boeck $2008^{21}$ & 62.7 & 57 & 54 & 188 & $A G$ vs $B M$ vs $B O$ \\
\hline Cascinu $2008^{22}$ & 64.0 & 65 & 73 & 84 & BT vs BR \\
\hline Wiedenmann $2008^{23}$ & 64.1 & 69 & 85 & 59 & Al vs AV \\
\hline Spano $2008^{24}$ & 63.7 & 102 & 57 & 103 & $\mathrm{AJ}$ vs $\mathrm{Al}$ \\
\hline Eckhardt $2009^{25}$ & 61.5 & 160 & 72 & 244 & $\mathrm{Bl}$ vs $\mathrm{Al}$ \\
\hline Poplin $2009^{26}$ & 63.5 & 96 & 90 & 547 & $A l$ vs $B O$ \\
\hline Saif $2009^{27}$ & 62.0 & 68 & 90 & 133 & $\mathrm{AY}$ vs $\mathrm{Al}$ \\
\hline Richards $201 \mathrm{I}^{28}$ & 66.9 & 67 & 89 & 130 & AP vs Al \\
\hline Cunningham $2009^{29}$ & 62.0 & 70 & 71 & 533 & Al vs BM \\
\hline Meyer $2010^{30}$ & 60.3 & 75 & 81 & 21 & $A I$ vs $B L$ \\
\hline Colucci $2010^{31}$ & 63.0 & 68 & 84 & 400 & $A I$ vs $B R$ \\
\hline Kindler $2010^{32}$ & 64.3 & 84 & 85 & 602 & $\mathrm{AK}$ vs $\mathrm{Al}$ \\
\hline Philip $2010^{33}$ & 64.0 & 91 & 79 & 743 & Al vs $A L$ \\
\hline Kindler $201 \mathrm{I}^{34}$ & 61.5 & 66 & 72 & 630 & $\mathrm{AJ}$ vs $\mathrm{Al}$ \\
\hline Reni $2012^{35}$ & 60.0 & 67 & 66 & 105 & $\mathrm{CG}$ vs $\mathrm{CH}$ \\
\hline Ko et al $2012^{17}$ & 63.8 & 81 & 77 & 61 & CK vs CJ \\
\hline Heinrich $2011^{36}$ & 61.0 & 64 & 75 & 151 & $\mathrm{BD}$ vs $\mathrm{Al}$ \\
\hline Löhr 2012 $2^{37}$ & 61.0 & 54 & 76 & 109 & $A I$ vs $B S$ \\
\hline Maraveyas $2012^{38}$ & 63.0 & 71 & 54 & 123 & Al vs BK \\
\hline Gilliam 201239 & 62.0 & 105 & 79 & 154 & $A F$ vs $C C$ \\
\hline Ozaka $2012^{40}$ & 64.0 & 67 & 72 & 116 & $A I$ vs $B V$ \\
\hline Nakai et al $2012^{30}$ & 65.0 & 41 & 74 & 106 & $A I$ vs $B V$ \\
\hline Gonçalves $2012^{41}$ & 62.5 & 68 & 80 & 104 & $A I$ vs $B F$ \\
\hline Meng $2012^{42}$ & 60.9 & 65 & 76 & 76 & AT vs Al \\
\hline Ueno et al $2013^{32}$ & 65.0 & 67 & 76 & 832 & $A l$ vs $C D$ vs $B V$ \\
\hline \multicolumn{6}{|l|}{ Okusaka et al $2017^{33}$} \\
\hline Hong $2014^{43}$ & 58.0 & 65 & 88 & 114 & $\mathrm{BE}$ vs $\mathrm{Al}$ \\
\hline
\end{tabular}


Table I (Continued)

\begin{tabular}{|c|c|c|c|c|c|}
\hline Study & $\begin{array}{l}\text { Mean or median } \\
\text { age }\end{array}$ & $\begin{array}{l}\text { Male/female } \\
\text { (ratio \%) }\end{array}$ & $\begin{array}{l}\text { Metastatic/advanced } \\
\text { (ratio \%) }\end{array}$ & $\begin{array}{l}\text { Total number } \\
\text { of participants }\end{array}$ & Comparison \\
\hline Cascinu $2014^{44}$ & 66.5 & 73 & 67 & 114 & $\mathrm{BU}$ vs $\mathrm{BR}$ \\
\hline Sudo et al $2014^{31}$ & 66.5 & 66 & 63 & 101 & $\mathrm{BV}$ vs $\mathrm{Al}$ \\
\hline Middleton et al $2014^{25}$ & 62.5 & 76 & 69 & 712 & $\mathrm{BM}$ vs $\mathrm{BN}$ \\
\hline Bergmann $2015^{45}$ & 63.3 & 86 & 72 & 106 & Al vs BG \\
\hline Borad 2015 $5^{46}$ & 66.0 & 74 & 76 & 143 & $\mathrm{Al}$ vs $\mathrm{BH}$ \\
\hline Bendell $2015^{47}$ & 65.3 & 100 & 82 & 22 & $\mathrm{BC}$ vs $\mathrm{Al}$ \\
\hline Deplanque $2015^{48}$ & 62.1 & 85 & 84 & 353 & $\mathrm{BA}$ vs $\mathrm{Al}$ \\
\hline Yamaue $2015^{49}$ & 64.0 & 65 & 73 & 153 & $\mathrm{AO}$ vs $\mathrm{Al}$ \\
\hline Kordes $2015^{50}$ & 64.5 & 98 & 74 & 121 & $A Q$ vs $A R$ \\
\hline Dalgleish $2016^{51}$ & 67.4 & 86 & 84 & 110 & $\mathrm{AU}$ vs $\mathrm{Al}$ \\
\hline Lee et al $2017^{26}$ & 54.0 & 78 & 72 & 214 & $A l$ vs $B M$ \\
\hline Laquente $2017^{52}$ & 64.3 & 60 & 77 & 99 & $\mathrm{AX}$ vs $\mathrm{Al}$ \\
\hline Middleton $2017^{53}$ & 67.0 & $14 \mid$ & 71 & 142 & $\mathrm{BJ}$ vs $\mathrm{Al}$ \\
\hline
\end{tabular}

Notes: An explanation of the two-letter codes is provided in Table 2.

Abbreviation: NA, not available.

Table 2 The chemotherapy regimens included in the study with the codes used in the figures

\begin{tabular}{|c|c|c|c|c|c|}
\hline Code & Chemotherapy regimen & Code & Chemotherapy regimen & Code & Chemotherapy regimen \\
\hline AA & $5-\mathrm{FU}$ & AV & Gemcitabine + infliximab & $\mathrm{BQ}$ & Gemcitabine+5-FU \\
\hline$A B$ & BAY I2-9566 & AW & Gemcitabine + irinotecan & BR & GemCis (gemcitabine + cisplatin) \\
\hline$A C$ & $\begin{array}{l}\text { FLEC (fluorouracil + } \\
\text { leucovorin }+ \text { epirubicin }+ \\
\text { carboplatin) }\end{array}$ & $A X$ & Gemcitabine+LY2603618 & BS & $\begin{array}{l}\text { Gemcitabine+ET (cationic liposomal } \\
\text { paclitaxel) }\end{array}$ \\
\hline$A D$ & Flutamide & AY & Gemcitabine+LY2931III & BT & $\begin{array}{l}\text { Gemcitabine+CDDP (cisplatin) + } \\
\text { cetuximab }\end{array}$ \\
\hline $\mathrm{AE}$ & 5-FU+MMC (mitomycin) & $A Z$ & Gemcitabine + marimastat & $\mathrm{BU}$ & $\begin{array}{l}\text { Gemcitabine+CDDP (cisplatin) + } \\
\text { sorfenib }\end{array}$ \\
\hline $\mathrm{AF}$ & GI7DT & BA & Gemcitabine + masitinib & BV & GS (gemcitabine+S-I) \\
\hline AG & $\begin{array}{l}\text { CapOx (capecitabine }+ \\
\text { oxaliplatin) }\end{array}$ & $\mathrm{BB}$ & Gemcitabine+NSC-63I570 & BW & Imatinib \\
\hline $\mathrm{AH}$ & Gastrazole & $\mathrm{BC}$ & Gemcitabine + olaparib & $\mathrm{BX}$ & NSC-63I570 \\
\hline Al & Gemcitabine & $\mathrm{BD}$ & Gemcitabine+RPIOI & BY & OXA (oxaliplatin) \\
\hline AJ & Gemcitabine + axitinib & $\mathrm{BE}$ & Gemcitabine + simvastatin & BZ & OXFU (5-FU+oxaliplatin) \\
\hline AK & Gemcitabine + bevacizumab & $\mathrm{BF}$ & Gemcitabine + sorafenib & CA & $\begin{array}{l}\text { PEFG (cisplatin + epirubicin + } \\
\text { fluorouracil + gemcitabine) }\end{array}$ \\
\hline $\mathrm{AL}$ & Gemcitabine + cetuximab & BG & SUNGEM (gemcitabine + sunitinib) & $\mathrm{CB}$ & PF (5-FU+cisplatin) \\
\hline AM & Gemcitabine+Cl-994 & $\mathrm{BH}$ & Gemcitabine+TH-302 & $\mathrm{CC}$ & Placebo \\
\hline AN & Gemcitabine + cilengitide & $\mathrm{BI}$ & Gemcitabine + tipifarnib & $C D$ & S-I \\
\hline $\mathrm{AO}$ & Gemcitabine + elpamotide & BJ & Gemcitabine + vandetanib & CE & ZD9331 \\
\hline $\mathrm{AP}$ & Gemcitabine + enzastaurin & BK & $\begin{array}{l}\text { GEMWAD (gemcitabine + weight- } \\
\text { adjusted dalteparin) }\end{array}$ & $\mathrm{CF}$ & Gemcitabine + docetaxel \\
\hline AQ & Gemcitabine + erlotinib & BL & Gemcitabine+Z-360 & CG & $\begin{array}{l}\text { PDXG (cisplatin + docetaxel + } \\
\text { capecitabine + gemcitabine) }\end{array}$ \\
\hline$A R$ & $\begin{array}{l}\text { Gemcitabine + erlotinib + } \\
\text { metformin }\end{array}$ & BM & $\begin{array}{l}\text { GemCape (gemcitabine }+ \\
\text { capecitabine) }\end{array}$ & $\mathrm{CH}$ & $\begin{array}{l}\text { PEXG (cisplatin + epirubicin + } \\
\text { capecitabine }+ \text { gemcitabine) }\end{array}$ \\
\hline AS & Gemcitabine + exatecan & $\mathrm{BN}$ & $\begin{array}{l}\text { GemCape (gemcitabine }+ \\
\text { capecitabine)+GVI00I+GM-CSF }\end{array}$ & $\mathrm{Cl}$ & CDDP (cisplatin) + docetaxel \\
\hline AT & Gemcitabine + huachansu & $\mathrm{BO}$ & $\begin{array}{l}\text { GemOX (gemcitabine }+ \\
\text { oxaliplatin) }\end{array}$ & CJ & Bevacizumab + cetuximab \\
\hline $\mathrm{AU}$ & Gemcitabine+IMM-I0I & $\mathrm{BP}$ & PG (gemcitabine + pemetrexed) & CK & $\begin{array}{l}\text { Gemcitabine + bevacizumab }+ \\
\text { cetuximab }\end{array}$ \\
\hline
\end{tabular}




\begin{tabular}{|c|c|c|c|c|c|c|c|c|c|c|c|c|c|c|}
\hline BX & $\begin{array}{c}0.97 \\
(0.41-2.30) \\
\end{array}$ & \begin{tabular}{c|}
0.70 \\
$0.34-1.42)$ \\
\end{tabular} & \begin{tabular}{|c|}
0.68 \\
$(0.33-1.37)$ \\
\end{tabular} & \begin{tabular}{|c|}
0.78 \\
$(0.27-2.28)$ \\
\end{tabular} & \begin{tabular}{|c|}
0.66 \\
$(0.32-1.36)$ \\
\end{tabular} & $\begin{array}{c}0.65 \\
(0.32-1.32) \\
\end{array}$ & $\begin{array}{c}0.57 \\
(0.30-1.09) \\
\end{array}$ & \begin{tabular}{|c|}
0.53 \\
$(0.30-0.95)$ \\
\end{tabular} & \begin{tabular}{|c|}
0.53 \\
$(0.29-0.96)$ \\
\end{tabular} & \begin{tabular}{|c|}
0.52 \\
$(0.29-0.94)$ \\
\end{tabular} & \begin{tabular}{c|}
0.52 \\
$(0.28-0.98)$ \\
\end{tabular} & \begin{tabular}{|c|}
0.51 \\
$(0.26-1.00)$ \\
\end{tabular} & $\begin{array}{c}0.44 \\
(0.24-0.76) \\
\end{array}$ & \begin{tabular}{|c|}
0.06 \\
$(0.02-0.24)$ \\
\end{tabular} \\
\hline CA & BB & $\begin{array}{c}0.72 \\
(0.33-1.55) \\
\end{array}$ & $\begin{array}{c}0.70 \\
(0.32-1.50)\end{array}$ & $\begin{array}{c}0.81 \\
(0.27-2.44) \\
\end{array}$ & $\begin{array}{c}0.68 \\
(0.31-1.48) \\
\end{array}$ & $\begin{array}{c}0.67 \\
(0.31-1.45) \\
\end{array}$ & $\begin{array}{c}0.58 \\
(0.29-1.20) \\
\end{array}$ & $\begin{array}{c}0.55 \\
(0.28-1.05) \\
\end{array}$ & $\begin{array}{c}0.54 \\
(0.28-1.06) \\
\end{array}$ & $\begin{array}{c}0.54 \\
(0.28-1.04)\end{array}$ & $\begin{array}{c}0.54 \\
(0.27-1.08) \\
\end{array}$ & $\begin{array}{c}0.52 \\
(0.25-1.09) \\
\end{array}$ & $\begin{array}{c}0.45 \\
(0.24-0.86 \\
\end{array}$ & \begin{tabular}{|l|}
0.07 \\
$.02-0.25)$ \\
\end{tabular} \\
\hline 0.86 & $\mathrm{BH}$ & CA & $\begin{array}{l}0.97 \\
.54-1.7\end{array}$ & $\begin{array}{c}1.12 \\
.42-3.04\end{array}$ & $\begin{array}{c}0.94 \\
.51-1.7\end{array}$ & $\begin{array}{l}0.93 \\
52-1.69\end{array}$ & 0.82 & $\begin{array}{l}0.76 \\
.50-1.18 \\
\end{array}$ & 0.76 & 0.75 & $\begin{array}{l}0.75 \\
46-1.23 \\
46\end{array}$ & 0.73 & 0.63 & 0.09 \\
\hline $\begin{array}{c}0.80 \\
(0.52-1.25) \\
\end{array}$ & $\begin{array}{c}0.94 \\
(0.62-1.41) \\
\end{array}$ & BV & $\mathrm{AC}$ & $\begin{array}{c}1.16 \\
(0.43-3.14) \\
\end{array}$ & $\begin{array}{c}0.97 \\
0.53-1.80) \\
\end{array}$ & $\begin{array}{c}0.96 \\
(0.53-1.74) \\
\end{array}$ & $\begin{array}{c}0.84 \\
(0.50-1.42) \\
\end{array}$ & \begin{tabular}{|c|}
0.79 \\
$(0.51-1.21)$ \\
\end{tabular} & $\begin{array}{c}0.78 \\
(0.50-1.24) \\
\end{array}$ & $\begin{array}{c}0.77 \\
(0.49-1.20) \\
\end{array}$ & $\begin{array}{c}0.77 \\
(0.47-1.27) \\
\end{array}$ & $\begin{array}{c}0.75 \\
(0.44-1.30) \\
\end{array}$ & & $\begin{array}{c}0.09 \\
(0.03-0.33) \\
\end{array}$ \\
\hline $\begin{array}{r}0.87 \\
47-1 .\end{array}$ & $\begin{array}{c}1.01 \\
.56-1.82) \\
\end{array}$ & $\begin{array}{l}1.08 \\
67-1\end{array}$ & $A U$ & BZ & $\begin{array}{c}0.84 \\
.31-2.30) \\
\end{array}$ & $\begin{array}{c}0.83 \\
(0.31-2.24) \\
\end{array}$ & $\begin{array}{c}0.73 \\
0.28-1.88) \\
\end{array}$ & $\begin{array}{c}0.68 \\
(0.27-1.68) \\
\end{array}$ & $\begin{array}{c}0.68 \\
0.27-1.69) \\
0\end{array}$ & $\begin{array}{c}0.66 \\
(0.27-1.66) \\
\end{array}$ & $\begin{array}{c}0.66 \\
(0.26-1.70) \\
\end{array}$ & $\begin{array}{c}0.65 \\
(0.25-1.71) \\
\end{array}$ & $\begin{array}{c}0.55 \\
0.22-1.36 \\
\end{array}$ & $\begin{array}{c}0.08 \\
02-0.36)\end{array}$ \\
\hline $\begin{array}{r}0.77 \\
(0.42-1 .\end{array}$ & $\begin{array}{c}0.89 \\
(0.50-1.58)\end{array}$ & $\begin{array}{c}0.95 \\
(0.61-1.49) \\
\end{array}$ & $\begin{array}{c}0.88 \\
(0.48-1.63)\end{array}$ & A) & B & $\begin{array}{c}0.99 \\
0.54-1.83) \\
\end{array}$ & $\begin{array}{c}0.86 \\
(0.50-1.49) \\
\end{array}$ & $\begin{array}{c}0.81 \\
(0.51-1.28 \\
\end{array}$ & $\begin{array}{c}0.80 \\
(0.50-1.30)\end{array}$ & $\begin{array}{c}0.79 \\
(0.49-1.27)\end{array}$ & $\begin{array}{c}0.79 \\
(0.47-1.33) \\
\end{array}$ & $\begin{array}{c}0.78 \\
(0.44-1.37) \\
\end{array}$ & $\begin{array}{c}0.67 \\
(0.43-1.04\end{array}$ & $\begin{array}{c}0.10 \\
(0.03-0.35) \\
\end{array}$ \\
\hline $\begin{array}{c}0.71 \\
.44-1.13) \\
\end{array}$ & $\begin{array}{c}0.82 \\
(0.53-1.28)\end{array}$ & $\begin{array}{c}0.88 \\
.68-1.14) \\
\end{array}$ & $\begin{array}{c}0.82 \\
(0.50-1.34)\end{array}$ & $\begin{array}{c}0.9 \\
.58-\end{array}$ & $B Q$ & AU & $\begin{array}{c}0.87 \\
0.52-1.47) \\
\end{array}$ & $\begin{array}{c}0.82 \\
(0.53-1.26)\end{array}$ & $\begin{array}{c}0.81 \\
(0.52-1.29)\end{array}$ & $\begin{array}{c}0.80 \\
(0.51-1.25)\end{array}$ & $\begin{array}{c}0.80 \\
(0.49-1.32) \\
\end{array}$ & $\begin{array}{c}0.78 \\
(0.45-1.35) \\
\end{array}$ & $\begin{array}{c}0.67 \\
0.44-1.02\end{array}$ & $\begin{array}{c}0.10 \\
.03-0.35) \\
\end{array}$ \\
\hline $\begin{array}{r}0.67 \\
0.42-1 .\end{array}$ & $\begin{array}{c}0.77 \\
(0.50-1.18)\end{array}$ & $\begin{array}{c}0.82 \\
0.65-1.05)\end{array}$ & $\begin{array}{c}0.76 \\
(0.47-1.24) \\
\end{array}$ & $\begin{array}{c}0.86 \\
(0.54-1.38)\end{array}$ & $\begin{array}{c}0.93 \\
(0.70-1.24)\end{array}$ & $A Q$ & AG & $\begin{array}{c}0.94 \\
(0.68-1.30)\end{array}$ & $\begin{array}{c}0.93 \\
(0.65-1.33\end{array}$ & $\begin{array}{c}0.92 \\
(0.65-1.29)\end{array}$ & $\begin{array}{c}0.92 \\
(0.61-1.38)\end{array}$ & $\begin{array}{c}0.90 \\
(0.56-1.43)\end{array}$ & $\begin{array}{c}0.77 \\
(0.57-1.06\end{array}$ & $\begin{array}{c}0.11 \\
(0.03-0.38) \\
(0.03\end{array}$ \\
\hline $\begin{array}{c}0.68 \\
0.37-1.23)\end{array}$ & $\begin{array}{c}0.79 \\
(0.44-1.40)\end{array}$ & $\begin{array}{c}0.84 \\
(0.53-1.33)\end{array}$ & $\begin{array}{c}0.78 \\
(0.42-1.45)\end{array}$ & $\begin{array}{c}0.89 \\
(0.48-1.62)\end{array}$ & $\begin{array}{c}0.96 \\
0.59-1.55)\end{array}$ & \begin{tabular}{|c|}
1.03 \\
$(0.64-1.64)$
\end{tabular} & BS & BM & \begin{tabular}{c|}
1.00 \\
$.81-1.23)$ \\
\end{tabular} & $\begin{array}{c}0.98 \\
(0.81-1.18)\end{array}$ & $\begin{array}{c}0.98 \\
(0.73-1.31) \\
\end{array}$ & $\begin{array}{c}0.96 \\
(0.67-1.38)\end{array}$ & $\begin{array}{c}0.82 \\
(0.74-0.92\end{array}$ & $\begin{array}{c}0.12 \\
(0.04-0.40)\end{array}$ \\
\hline $\begin{array}{c}0.62 \\
0.41-0.95)\end{array}$ & $\begin{array}{c}0.72 \\
0.49-1.0\end{array}$ & $\begin{array}{c}0.77 \\
.65-0.92) \\
\end{array}$ & $\begin{array}{c}0.71 \\
(0.45-1.13)\end{array}$ & $\begin{array}{c}0.81 \\
(0.52-1.25)\end{array}$ & \begin{tabular}{|c|}
0.88 \\
$(0.69-1.11)$
\end{tabular} & $\begin{array}{c}0.94 \\
(0.76-1.1\end{array}$ & $\begin{array}{r}0.0 \\
(0.59- \\
\end{array}$ & BM & $A Q$ & $\begin{array}{c}0.98 \\
(0.78-1.25)\end{array}$ & $\begin{array}{c}0.98 \\
(0.71-1.36) \\
\end{array}$ & $\begin{array}{c}0.96 \\
(0.65-1.43)\end{array}$ & 0.83 & $\begin{array}{c}0.12 \\
(0.04-0.40)\end{array}$ \\
\hline \begin{tabular}{c|}
0.59 \\
$(0.38-0.91)$
\end{tabular} & $\begin{array}{c}0.69 \\
(0.46-1.03)\end{array}$ & $\begin{array}{c}0.74 \\
(0.60-0.90)\end{array}$ & \begin{tabular}{|c|}
0.68 \\
$(0.43-1.08)$
\end{tabular} & $\begin{array}{c}0.77 \\
(0.50-1.21)\end{array}$ & \begin{tabular}{c|}
0.83 \\
$(0.65-1.07)$
\end{tabular} & \begin{tabular}{c|}
0.90 \\
$(0.71-1.13)$
\end{tabular} & $\begin{array}{c}0.87 \\
(0.56-1.37)\end{array}$ & $\begin{array}{c}0.89 \\
(0.71-1.13)\end{array}$ & BO & BV & $\begin{array}{c}1.00 \\
(0.74-1.36)\end{array}$ & $\begin{array}{c}0.98 \\
(0.67-1.44)\end{array}$ & $\begin{array}{c}0.84 \\
.73-0.98\end{array}$ & \begin{tabular}{|l|}
0.12 \\
$.04-0.41)$
\end{tabular} \\
\hline $\begin{array}{c}0.58 \\
(0.31-1.10)\end{array}$ & $\begin{array}{c}0.68 \\
(0.37-1.26)\end{array}$ & $\begin{array}{c}0.72 \\
(0.44-1.20)\end{array}$ & $\begin{array}{c}0.67 \\
(0.35-1.30)\end{array}$ & $\begin{array}{c}0.76 \\
(0.40-1.45)\end{array}$ & $\begin{array}{c}0.82 \\
(0.48-1.40)\end{array}$ & $\begin{array}{c}0.88 \\
(0.52-1.48)\end{array}$ & $\begin{array}{c}0.86 \\
(0.45-1.6\end{array}$ & $\begin{array}{c}0.88 \\
(0.52-1.48)\end{array}$ & $\begin{array}{r}0.9 \\
(0.60-\end{array}$ & BT & BN & $\begin{array}{c}0.98 \\
0.63-1.52)\end{array}$ & $\begin{array}{c}0.84 \\
0.65-1.1\end{array}$ & $\begin{array}{c}0.12 \\
(0.04-0.42)\end{array}$ \\
\hline $\begin{array}{c}0.58 \\
(0.34-1.00)\end{array}$ & $\begin{array}{c}0.68 \\
(0.40-1.14)\end{array}$ & $\begin{array}{c}0.74 \\
(0.50-1.06)\end{array}$ & $\begin{array}{c}0.67 \\
(0.38-1.18)\end{array}$ & $\begin{array}{c}0.76 \\
(0.44-1.32) \\
\end{array}$ & $\begin{array}{c}0.82 \\
0.55-1.24) \\
\end{array}$ & $\begin{array}{c}0.88 \\
.59-1.31) \\
\end{array}$ & $\begin{array}{c}0.86 \\
(0.49-1.49) \\
\end{array}$ & \begin{tabular}{c|}
0.88 \\
$0.59-1.31)$ \\
\end{tabular} & \begin{tabular}{c|}
0.98 \\
$0.68-1.43)$ \\
\end{tabular} & $\begin{array}{r}1.00 \\
.55-1\end{array}$ & AP & $\mathrm{BH}$ & $\begin{array}{c}0.86 \\
(0.61-1.22\end{array}$ & $\begin{array}{c}0.12 \\
0.04-0.43) \\
0.04\end{array}$ \\
\hline $\begin{array}{c}0.58 \\
(0.35-0.96) \\
\end{array}$ & $\begin{array}{c}0.67 \\
(0.42-1.08)\end{array}$ & $\begin{array}{c}0.72 \\
.52-0.99) \\
\end{array}$ & \begin{tabular}{|c|}
0.66 \\
$(0.39-1.13)$ \\
\end{tabular} & \begin{tabular}{|c|}
0.75 \\
$(0.45-1.26)$ \\
\end{tabular} & \begin{tabular}{|c|}
0.81 \\
$(0.57-1.17)$ \\
\end{tabular} & $\begin{array}{c}0.87 \\
(0.60-1.28) \\
\end{array}$ & $\begin{array}{c}0.85 \\
(0.51-1.43) \\
\end{array}$ & $\begin{array}{c}0.87 \\
(0.62-1.23) \\
\end{array}$ & $\begin{array}{c}0.98 \\
(0.71-1.34) \\
\end{array}$ & $\begin{array}{c}0.99 \\
(0.57-1.74) \\
\end{array}$ & & $A C$ & $\mathrm{Al}$ & $\begin{array}{c}0.14 \\
.04-0.47) \\
\end{array}$ \\
\hline $\begin{array}{c}0.51 \\
.34-0.77) \\
\end{array}$ & $\begin{array}{c}0.59 \\
(0.40-0.87)\end{array}$ & \begin{tabular}{c|}
0.63 \\
$0.55-0.74)$ \\
\end{tabular} & $\begin{array}{c}0.58 \\
(0.38-0.92)\end{array}$ & \begin{tabular}{|c|}
0.66 \\
$(0.43-1.0$ \\
\end{tabular} & $\begin{array}{c}0.71 \\
0.57-0.8\end{array}$ & $\begin{array}{c}0.77 \\
0.50-1.23)\end{array}$ & $\begin{array}{c}0.75 \\
0.49-1.1\end{array}$ & \begin{tabular}{c|}
0.77 \\
$0.63-0.92)$ \\
\end{tabular} & $\begin{array}{c}0.86 \\
0.75-0.97)\end{array}$ & $\begin{array}{c}0.87 \\
0.53-1.3\end{array}$ & $\begin{array}{c}0.87 \\
0.62-1.24\end{array}$ & 0.8 & $\mathrm{Al}$ & CC \\
\hline $\begin{array}{l}0.27 \\
17-0.4\end{array}$ & $\begin{array}{c}0.31 \\
(0.20-1.49)\end{array}$ & $\begin{array}{c}0.33 \\
(0.25-0.4\end{array}$ & $\begin{array}{c}0.31 \\
(0.19-0.52)\end{array}$ & $\begin{array}{c}0.35 \\
(0.21-0.57)\end{array}$ & $\begin{array}{c}0.38 \\
(0.27-1.53\end{array}$ & $\begin{array}{c}0.41 \\
(0.30-1.5\end{array}$ & $\begin{array}{r}0.40 \\
0.24-0 .\end{array}$ & $\begin{array}{l}0.41 \\
.30-0.5\end{array}$ & $\begin{array}{c}0.45 \\
34-0.6\end{array}$ & $\begin{array}{c}0.46 \\
(0.27-0.8\end{array}$ & $\begin{array}{c}0.46 \\
0.30-0.7\end{array}$ & $\begin{array}{c}0.46 \\
0.32-0.6\end{array}$ & $\begin{array}{c}0.53 \\
0.41-0.68)\end{array}$ & AE \\
\hline
\end{tabular}

Overall survival:

Regimen

$\mathrm{HR}(95 \% \mathrm{Crl})$

Progression-free survival:

Regimen

$\mathrm{HR}(95 \% \mathrm{Crl})$

Figure 3 Selected results of the random-effects model network meta-analyses of overall survival and progression-free survival.

Notes: The treatment regimens are listed in order of overall survival and progression-free survival ranked according to the SUCRA. It lists only the top I 3 chemotherapy regimens (by SUCRA), and it also includes gemcitabine and the least useful regimen as references. Comparisons should be read from left to right. The intersection of the column-defining chemotherapy regimen and the row-defining regimen shows the HRs of overall survival and progression-free survival. For overall survival, an HR below I favors the row-defining regimen. For progression-free survival, an HR below I favors the column-defining regimen. Reciprocals of corresponding HRs should be taken to get HRs for comparison in the opposing direction. An explanation of the two-letter codes is provided in Table 2.

Abbreviations: $\mathrm{Crl}$, credible interval; HR, hazard ratio; SUCRA, surface under the cumulative ranking curve.

\begin{tabular}{|c|c|c|c|c|c|c|c|c|c|c|c|c|c|c|}
\hline CA & $\begin{array}{c}0.69 \\
.02-9.30)\end{array}$ & $\begin{array}{l}1.29 \\
.04-15.71) \\
\end{array}$ & $(0.51-9.76)$ & \begin{tabular}{c|}
2.30 \\
$(0.38-14.56)$ \\
\end{tabular} & $\begin{array}{c}2.49 \\
(0.56-12.67) \\
\end{array}$ & $\begin{array}{c}2.29 \\
(0.29-17.17) \\
\end{array}$ & $\begin{array}{c}1.80 \\
(0.04-32.74) \\
\end{array}$ & $\begin{array}{c}2.69 \\
(0.49-16.24) \\
\end{array}$ & $\begin{array}{c}2.00 \\
(0.04-44.98) \\
\end{array}$ & $\begin{array}{c}2.67 \\
(0.35-21.02) \\
\end{array}$ & \begin{tabular}{c|}
2.82 \\
$(0.42-19.97)$ \\
\end{tabular} & $\begin{array}{c}3.13 \\
(0.70-16.66) \\
\end{array}$ & \begin{tabular}{|c|}
7.16 \\
$(2.04-31.76)$ \\
\end{tabular} & $\begin{array}{c}61.09 \\
(5.27-2818.49) \\
\end{array}$ \\
\hline BX & BB & $\begin{array}{c}1.84 \\
04-95.42) \\
\end{array}$ & 3.91) & 14) & 5) & $(0$. & 2 & 50) & .89) & $0.15)$ & 4. & $2.60)$ & & 6.21 \\
\hline$(0.12-10.20)$ & CA & $\mathrm{AU}$ & $\begin{array}{r}1.59 \\
20-44\end{array}$ & $\begin{array}{r}1.7 \\
17-5\end{array}$ & $\begin{array}{r}1 . \\
0.22-\end{array}$ & $\begin{array}{r}1 . \\
(0.13-\end{array}$ & $\begin{array}{r}1 . \\
(0.02-\end{array}$ & $\begin{array}{r}2 \\
(0.20 \\
\end{array}$ & $\left(\begin{array}{r}1 . \\
(0.03-\end{array}\right.$ & $\begin{array}{r}2 . \\
(0.16- \\
\end{array}$ & $\begin{array}{r}2 . \\
(0.18-\end{array}$ & $\begin{array}{r}2 \\
(0.28- \\
\end{array}$ & $3.12)$ & $\begin{array}{r}53 \\
2.37-6\end{array}$ \\
\hline \begin{tabular}{c|}
2.72 \\
$(0.48-19.54)$ \\
\end{tabular} & $\begin{array}{c}1.63 \\
.42-6.71)\end{array}$ & BV & BV & \begin{tabular}{l|}
1.10 \\
$.30-3.77)$ \\
\end{tabular} & \begin{tabular}{c|}
1.19 \\
$.48-3.15)$ \\
\end{tabular} & $\begin{array}{c}1.09 \\
0.20-4.99) \\
\end{array}$ & $\begin{array}{c}0.86 \\
(0.02-10.65)\end{array}$ & $\begin{array}{c}1.30 \\
(0.39-4.37) \\
\end{array}$ & \begin{tabular}{|c|}
0.95 \\
$(0.02-14.89)$ \\
\end{tabular} & $\begin{array}{r}1.2 \\
(0.25- \\
\end{array}$ & $\begin{array}{r}1.3 \\
(0.29- \\
\end{array}$ & $\begin{array}{r}1.5 \\
(0.57 \\
\end{array}$ & $\begin{array}{r}3.4 \\
(2.11- \\
\end{array}$ & $\begin{array}{r}28 \\
(3.34-\end{array}$ \\
\hline \begin{tabular}{c|}
1.84 \\
$(0.14-28.44)$ \\
\end{tabular} & $\begin{array}{c}1.10 \\
11-11.00)\end{array}$ & $\begin{array}{c}0.68 \\
.09-4.96\end{array}$ & $\mathrm{BU}$ & AJ & & 1.6 & 37) & $94)$ & \begin{tabular}{c|}
0.87 \\
$(0.02-15.92)$ \\
\end{tabular} & $\begin{array}{r}1 . \\
(0.17 \\
\end{array}$ & $\begin{array}{r}1.2 \\
(0.20- \\
\end{array}$ & $\begin{array}{r}1.3 \\
(0.33- \\
\end{array}$ & $\begin{array}{r}3 . \\
(1.06- \\
\end{array}$ & $\begin{array}{r}26 \\
(2.42-\end{array}$ \\
\hline \begin{tabular}{|c|}
3.25 \\
$55-25.60)$ \\
\end{tabular} & $\begin{array}{c}1.94 \\
.46-9.0 \\
\end{array}$ & $\begin{array}{l}1.20 \\
51-2.89) \\
\end{array}$ & 1.7 & BR & $A W$ & $\begin{array}{c}0.92 \\
0.15-4.47) \\
\end{array}$ & $\begin{array}{c}0.71 \\
.02-9.57) \\
\end{array}$ & $\begin{array}{c}1.09 \\
(0.29-3.88) \\
\end{array}$ & \begin{tabular}{c|}
0.80 \\
$(0.02-12.83)$ \\
\end{tabular} & $\begin{array}{r}1 . \\
(0.18- \\
\end{array}$ & $\begin{array}{r}1.1 \\
(0.23- \\
\end{array}$ & $\begin{array}{r}1.3 \\
(0.41- \\
\end{array}$ & $\begin{array}{r}2.8 \\
(1.36- \\
\end{array}$ & $\begin{array}{r}23 \\
(2.62- \\
\end{array}$ \\
\hline \begin{tabular}{c|}
3.49 \\
$0.51-29.58)$ \\
\end{tabular} & $\begin{array}{r}2.1 \\
43-1 \\
\end{array}$ & $\begin{array}{c}1.28 \\
.44-3.71) \\
\end{array}$ & $\begin{array}{c}1.90 \\
.22-15.75) \\
\end{array}$ & $\begin{array}{r}1.0 \\
(0.32-3 \\
\end{array}$ & $\mathrm{BN}$ & $A X$ & .02-14.99) & & .51) & $\begin{array}{r}1.1 \\
(0.14-1 \\
\end{array}$ & $\begin{array}{r}1.2 \\
(0.16- \\
\end{array}$ & $\begin{array}{r}1.3 \\
(0.27- \\
\end{array}$ & $\begin{array}{r}3.1 \\
(0.79- \\
\end{array}$ & $1.84)$ \\
\hline \begin{tabular}{|c|}
2.39 \\
$(0.04-81.10)$ \\
\end{tabular} & \begin{tabular}{|c|}
1.43 \\
$(0.03-34.97)$ \\
\end{tabular} & $\begin{array}{c}0.87 \\
02-17.04) \\
\end{array}$ & $\begin{array}{c}1.24 \\
0.02-45.58) \\
\end{array}$ & \begin{tabular}{|c|}
0.72 \\
$(0.01-14.93)$ \\
\end{tabular} & $\begin{array}{r}0.6 \\
(0.01-1 \\
\end{array}$ & BZ & AT & $\begin{array}{c}1.51 \\
.10-62.52) \\
\end{array}$ & \begin{tabular}{|c|}
1.10 \\
$(0.01-93.20)$ \\
\end{tabular} & \begin{tabular}{|c|}
1.53 \\
$(0.08-66.61)$ \\
\end{tabular} & \begin{tabular}{c|}
1.63 \\
$(0.09-68.45)$ \\
\end{tabular} & $\begin{array}{c}1.73 \\
(0.13-65.01) \\
\end{array}$ & $\begin{array}{c}4.06 \\
(0.34-143.04) \\
\end{array}$ & $\begin{array}{c}36.91 \\
(1.18-6038.65) \\
\end{array}$ \\
\hline \begin{tabular}{c|}
3.78 \\
$(0.51-35.08)$ \\
\end{tabular} & $\begin{array}{c}2.27 \\
(0.42-13.15) \\
\end{array}$ & $\begin{array}{c}1.38 \\
40-4.74) \\
\end{array}$ & $\begin{array}{c}2.04 \\
.22-18.82) \\
\end{array}$ & $\begin{array}{c}1.15 \\
.30-4.36) \\
\end{array}$ & \begin{tabular}{c|}
1.07 \\
$(0.26-4.63)$ \\
\end{tabular} & \begin{tabular}{|c|}
1.62 \\
$(0.07-90.07)$ \\
\end{tabular} & $\mathrm{BF}$ & $\mathrm{BH}$ & \begin{tabular}{c|}
0.74 \\
$(0.02-13.00)$ \\
\end{tabular} & $\begin{array}{c}0.98 \\
(0.15-6.42) \\
\end{array}$ & $\begin{array}{c}1.05 \\
(0.18-6.09) \\
\end{array}$ & $\begin{array}{c}1.18 \\
(0.31-4.60) \\
\end{array}$ & $\begin{array}{c}2.68 \\
(0.95-8.22) \\
\end{array}$ & \begin{tabular}{c|}
22.77 \\
$(2.02-1006.14)$ \\
\end{tabular} \\
\hline $\begin{array}{c}3.89 \\
(0.55-35.09)\end{array}$ & $\begin{array}{c}2.37 \\
(0.43-13.39)\end{array}$ & $\begin{array}{l}1.44 \\
.42-4.87)\end{array}$ & $\begin{array}{c}2.09 \\
.23-19.69)\end{array}$ & $\begin{array}{c}1.21 \\
(0.31-4.45)\end{array}$ & $\begin{array}{c}1.12 \\
(0.25-4.84) \\
\end{array}$ & \begin{tabular}{c|}
1.68 \\
$(0.07-92.61)$
\end{tabular} & $\begin{array}{r}1.0 \\
(0.20\end{array}$ & AY & $B X$ & \begin{tabular}{c|}
1.35 \\
$(0.06-77.69)$
\end{tabular} & $\begin{array}{r}1 . \\
(0.07-\end{array}$ & $\begin{array}{c}1.59 \\
(0.10-71.44)\end{array}$ & $\begin{array}{c}3.64 \\
(0.25-148.70)\end{array}$ & $\begin{array}{r}34 \\
(0.88-5\end{array}$ \\
\hline $\begin{array}{c}3.80 \\
(0.37-41.93) \\
\end{array}$ & $\begin{array}{c}2.28 \\
29-17.67)\end{array}$ & $\begin{array}{c}1.39 \\
.26-7.23)\end{array}$ & $\begin{array}{c}2.05 \\
.20-20.37) \\
\end{array}$ & \begin{tabular}{c|}
1.16 \\
$.28-4.54)$ \\
\end{tabular} & $\begin{array}{c}1.07 \\
.17-6.48) \\
\end{array}$ & \begin{tabular}{|c|}
1.59 \\
$(0.06-96.54)$
\end{tabular} & $\begin{array}{c}1.00 \\
(0.14-6.80)\end{array}$ & $\begin{array}{r}0.9 \\
(0.14- \\
\end{array}$ & BT & BT & $\begin{array}{c}1.07 \\
(0.13-8.99) \\
\end{array}$ & $\begin{array}{c}1.19 \\
(0.21-6.82) \\
\end{array}$ & \begin{tabular}{|c|}
2.72 \\
$(0.62-12.92)$ \\
\end{tabular} & $\begin{array}{r}23 \\
(1.74-1 \\
\end{array}$ \\
\hline $\begin{array}{c}3.93 \\
(0.49-37.77)\end{array}$ & $\begin{array}{l}2.34 \\
39-15.06)\end{array}$ & $\begin{array}{l}1.45 \\
35-5.58)\end{array}$ & $\begin{array}{c}2.11 \\
.20-22.08)\end{array}$ & $\begin{array}{c}1.21 \\
0.27-5.14)\end{array}$ & \begin{tabular}{|c|}
1.13 \\
$(0.22-5.39)$ \\
\end{tabular} & $\begin{array}{c}1.70 \\
(0.07-100.60)\end{array}$ & $\begin{array}{c}1.04 \\
(0.18-5.62) \\
\end{array}$ & $\begin{array}{c}0.99 \\
(0.17-5.58) \\
\end{array}$ & $(0.13-$ & AW & $A C$ & 1) & \begin{tabular}{c|}
2.55 \\
$(0.69-11.07)$ \\
\end{tabular} & $\begin{array}{r}21 \\
(1.68-1 \\
\end{array}$ \\
\hline $\begin{array}{c}4.20 \\
(0.71-31.38)\end{array}$ & $\begin{array}{l}2.51 \\
.62-11.10)\end{array}$ & $\begin{array}{c}1.54 \\
.70-3.44)\end{array}$ & $\begin{array}{c}2.24 \\
.29-18.01)\end{array}$ & $\begin{array}{c}1.28 \\
(0.49-3.38)\end{array}$ & $\begin{array}{c}1.20 \\
(0.56-2.48)\end{array}$ & \begin{tabular}{|c|}
1.77 \\
$(0.09-88.53)$ \\
\end{tabular} & $\begin{array}{c}1.12 \\
(0.31-3.94)\end{array}$ & $\begin{array}{c}1.06 \\
(0.30-3.93)\end{array}$ & $\begin{array}{c}1.11 \\
(0.21-5.96)\end{array}$ & \begin{tabular}{|c|}
1.06 \\
$(0.26-4.52)$
\end{tabular} & B & BF & $\begin{array}{c}2.30 \\
(1.02-5.39)\end{array}$ & \begin{tabular}{|c|}
18.95 \\
$(2.04-777.60)$
\end{tabular} \\
\hline $\begin{array}{c}4.09 \\
(0.44-43.25) \\
\end{array}$ & $\begin{array}{c}2.40 \\
(0.37-16.18) \\
\end{array}$ & $\begin{array}{c}1.47 \\
.35-6.21) \\
\end{array}$ & $\begin{array}{c}2.21 \\
(0.20-23.44) \\
\end{array}$ & $\begin{array}{c}1.23 \\
(0.26-5.75) \\
\end{array}$ & $\begin{array}{c}1.15 \\
(0.22-6.09) \\
\end{array}$ & $\begin{array}{c}1.71 \\
(0.06-106.30) \\
\end{array}$ & $\begin{array}{c}1.08 \\
(0.18-6.19) \\
\end{array}$ & $\begin{array}{c}1.02 \\
(0.17-6.33) \\
\end{array}$ & $\begin{array}{c}1.08 \\
(0.14-8.72) \\
\end{array}$ & $\begin{array}{c}1.03 \\
(0.16-6.77) \\
\end{array}$ & $\begin{array}{c}0.97 \\
(0.22-4.38) \\
\end{array}$ & $B G$ & $\mathrm{Al}$ & $9.70)$ \\
\hline $\begin{array}{c}7.10 \\
1.41-49.70) \\
\end{array}$ & $\begin{array}{c}4.27 \\
(1.24-16.90) \\
\end{array}$ & $\begin{array}{c}2.65 \\
(1.66-4.38) \\
\end{array}$ & $\begin{array}{c}3.89 \\
(0.58-28.03) \\
\end{array}$ & $\begin{array}{c}2.20 \\
(1.08-4.61) \\
\end{array}$ & \begin{tabular}{c|}
2.05 \\
$(0.82-5.47)$ \\
\end{tabular} & $\begin{array}{c}3.07 \\
(0.16-154.61) \\
\end{array}$ & $\begin{array}{c}1.92 \\
(0.63-5.92) \\
\end{array}$ & $\begin{array}{c}1.84 \\
(0.61-5.96) \\
\end{array}$ & $\begin{array}{c}1.90 \\
(0.40-9.38) \\
\end{array}$ & $\begin{array}{c}1.83 \\
(0.52-6.97) \\
\end{array}$ & $\begin{array}{c}1.73 \\
(0.96-3.22) \\
\end{array}$ & $(0.46-7.12)$ & $\mathrm{Al}$ & B \\
\hline $\begin{array}{r}26.37 \\
66-250.8 \\
\end{array}$ & $\begin{array}{c}15.73 \\
.87-93.28) \\
\end{array}$ & $\begin{array}{c}9.50 \\
99-33.80)\end{array}$ & \begin{tabular}{|c|}
14.06 \\
$(1.46-133.96)$ \\
\end{tabular} & \begin{tabular}{|c|}
7.84 \\
$(2.16-31.80)$ \\
\end{tabular} & \begin{tabular}{|c|}
7.33 \\
$(1.74-34.15)$ \\
\end{tabular} & $\begin{array}{c}11.16 \\
(0.49-643.92) \\
\end{array}$ & $\begin{array}{c}6.80 \\
(1.45-33.67)\end{array}$ & \begin{tabular}{|c|}
6.63 \\
$(1.42-32.97)$ \\
\end{tabular} & \begin{tabular}{c|}
6.89 \\
$(1.06-47.78)$
\end{tabular} & $\begin{array}{c}6.66 \\
(1.22-37.61)\end{array}$ & $\begin{array}{c}6.18 \\
(1.78-22.84)\end{array}$ & $\begin{array}{c}6.43 \\
(1.14-38.99) \\
\end{array}$ & \begin{tabular}{c|}
3.59 \\
$(1.21-11.39)$ \\
\end{tabular} & $A B$ \\
\hline
\end{tabular}

Overall response rate: $\square$ Regimen $\square$ OR $(95 \% \mathrm{Crl}) \quad$ Disease control rate: $\square$ Regimen $\quad \square \quad$ OR (95\% Crl)

Figure 4 Selected results of network meta-analysis of overall response rate and disease control rate.

Notes: The treatment regimens are listed in order of overall response rate and disease control rate ranked according to SUCRA. The figure lists only the top 13 chemotherapy regimens (by SUCRA), and it also includes gemcitabine and the least useful regimen as references. Comparisons should be read from left to right. The intersection of the column-defining chemotherapy regimen and the row-defining regimen shows the ORs of overall response rate and disease control rate. For overall response rate, an OR value below I favors the column-defining treatment. For disease control rate, an OR value below I favors the row-defining treatment. Reciprocals of corresponding ORs should be taken to get ORs for comparison in the opposing direction. An explanation of the two-letter codes is provided in Table 2 .

Abbreviations: $\mathrm{Crl}$, credible interval; OR, odds ratio; SUCRA, surface under the cumulative ranking curve. 


\begin{tabular}{|c|c|c|c|c|c|c|c|c|c|c|c|c|c|c|}
\hline \multirow[t]{15}{*}{ BP } & $\begin{array}{c}1.06 \\
(0.17-4.81) \\
\end{array}$ & $\begin{array}{c}0.60 \\
(0.00-10.27) \\
\end{array}$ & $\begin{array}{c}1.50 \\
(0.43-4.93) \\
\end{array}$ & $\begin{array}{c}1.44 \\
(0.34-5.78) \\
\end{array}$ & $\begin{array}{c}1.29 \\
(0.19-8.06) \\
\end{array}$ & $\begin{array}{c}1.69 \\
(0.43-6.21) \\
\end{array}$ & $\begin{array}{c}1.83 \\
(0.58-5.80) \\
\end{array}$ & $\begin{array}{c}1.96 \\
(0.82-4.66) \\
\end{array}$ & $\begin{array}{c}1.94 \\
(0.69-5.42) \\
\end{array}$ & $\begin{array}{c}2.03 \\
(0.54-7.51) \\
\end{array}$ & $\begin{array}{c}2.19 \\
(0.53-8.30) \\
\end{array}$ & $\begin{array}{c}2.23 \\
(0.65-7.38) \\
\end{array}$ & \begin{tabular}{|c|c}
4.68 \\
$(2.24-9.64)$ \\
\end{tabular} & $\begin{array}{c}135.67 \\
(9.45-3203.07) \\
\end{array}$ \\
\hline & $A C$ & $\begin{array}{c}0.58 \\
(0.00-13.34)\end{array}$ & $\begin{array}{c}1.47 \\
(0.27-10.09) \\
\end{array}$ & \begin{tabular}{c|}
1.44 \\
$(0.22-10.41)$ \\
\end{tabular} & \begin{tabular}{|c|}
1.26 \\
$(0.13-12.98)$ \\
\end{tabular} & $\begin{array}{c}1.63 \\
(0.29-12.50) \\
\end{array}$ & $\begin{array}{c}1.75 \\
(0.35-12.19) \\
\end{array}$ & $\begin{array}{c}1.89 \\
(0.45-10.62) \\
\end{array}$ & $\begin{array}{c}1.87 \\
(0.39-11.49)\end{array}$ & $\begin{array}{c}1.98 \\
(0.35-14.28) \\
\end{array}$ & $\begin{array}{c}2.11 \\
(0.34-15.05) \\
\end{array}$ & $\begin{array}{c}2.15 \\
(0.39-14.32) \\
\end{array}$ & $\begin{array}{c}4.52 \\
(1.14-24.00) \\
\end{array}$ & $\begin{array}{c}135.10 \\
(6.59-4101.00) \\
\end{array}$ \\
\hline & & CE & $\begin{array}{c}2.54 \\
(0.13-433.10)\end{array}$ & $\begin{array}{c}2.43 \\
(0.12-403.80) \\
\end{array}$ & $\begin{array}{c}2.24 \\
0.08-401.00) \\
\end{array}$ & $\begin{array}{c}2.90 \\
(0.14-485.50) \\
\end{array}$ & $\begin{array}{c}3.03 \\
(0.17-528.80) \\
\end{array}$ & $\begin{array}{c}3.26 \\
(0.20-564.50) \\
\end{array}$ & $\begin{array}{c}3.29 \\
0.19-540.00) \\
\end{array}$ & $\begin{array}{c}3.48 \\
(0.18-611.00)\end{array}$ & $\begin{array}{c}3.69 \\
(0.19-661.60)\end{array}$ & $\begin{array}{c}3.81 \\
0.20-584.40) \\
\end{array}$ & $\begin{array}{c}7.81 \\
0.50-1301.41\end{array}$ & $\begin{array}{c}261.30 \\
(4.83-75950.00) \\
\end{array}$ \\
\hline & & & $\mathrm{BH}$ & \begin{tabular}{|c|}
0.97 \\
$(0.20-4.54)$ \\
\end{tabular} & \begin{tabular}{|c|}
0.86 \\
$(0.11-6.18)$ \\
\end{tabular} & \begin{tabular}{|c|}
1.13 \\
$(0.25-5.13)$ \\
\end{tabular} & $\begin{array}{c}1.21 \\
(0.32-4.62) \\
\end{array}$ & $\begin{array}{c}1.31 \\
(0.46-3.92) \\
\end{array}$ & $\begin{array}{c}1.29 \\
(0.38-4.44) \\
\end{array}$ & $\begin{array}{c}1.36 \\
(0.31-6.13) \\
\end{array}$ & $\begin{array}{c}1.45 \\
(0.32-6.52) \\
\end{array}$ & \begin{tabular}{|c|}
1.47 \\
$(0.38-6.01)$ \\
\end{tabular} & \begin{tabular}{|c|}
3.12 \\
$(1.21-8.61)$ \\
\end{tabular} & $\begin{array}{c}92.61 \\
(5.82-2300.44) \\
\end{array}$ \\
\hline & & & & CA & \begin{tabular}{|c|}
0.90 \\
$(0.11-7.30)$ \\
\end{tabular} & $\begin{array}{c}1.19 \\
(0.23-6.23) \\
\end{array}$ & \begin{tabular}{|c|}
1.26 \\
$(0.28-6.04)$ \\
\end{tabular} & $\begin{array}{c}1.36 \\
(0.38-5.22) \\
\end{array}$ & $\begin{array}{c}1.35 \\
(0.33-5.77) \\
\end{array}$ & $\begin{array}{c}1.41 \\
(0.28-7.36) \\
\end{array}$ & $\begin{array}{c}1.50 \\
(0.27-8.48) \\
\end{array}$ & \begin{tabular}{|c|}
1.54 \\
$(0.33-7.58)$ \\
\end{tabular} & $\begin{array}{c}3.24 \\
(1.00-11.60) \\
\end{array}$ & $\begin{array}{c}92.15 \\
(5.08-2606.88) \\
\end{array}$ \\
\hline & & & & & BT & \begin{tabular}{|c|}
1.33 \\
$(0.17-10.71)$ \\
\end{tabular} & $\begin{array}{c}1.40 \\
(0.22-10.49)\end{array}$ & $\begin{array}{c}1.52 \\
(0.28-9.53)\end{array}$ & $\begin{array}{c}1.51 \\
(0.24-10.73)\end{array}$ & $\begin{array}{c}1.58 \\
(0.21-12.90)\end{array}$ & $\begin{array}{c}1.68 \\
(0.23-14.01)\end{array}$ & $\begin{array}{c}1.72 \\
(0.25-13.66)\end{array}$ & $\begin{array}{c}3.61 \\
(0.70-21.92)\end{array}$ & $\begin{array}{c}111.67 \\
(4.55-3502.63) \\
\end{array}$ \\
\hline & & & & & & BG & $\begin{array}{c}1.07 \\
(0.25-4.45)\end{array}$ & $\begin{array}{c}1.15 \\
(0.35-4.15)\end{array}$ & $\begin{array}{c}1.15 \\
(0.30-4.54)\end{array}$ & $\begin{array}{c}1.19 \\
(0.25-5.84)\end{array}$ & $\begin{array}{c}1.27 \\
(0.25-6.31)\end{array}$ & \begin{tabular}{|c|}
1.33 \\
$(0.30-5.86)$
\end{tabular} & $\begin{array}{c}2.74 \\
(0.92-8.95) \\
\end{array}$ & $\begin{array}{c}82.03 \\
(5.00-2015.32) \\
\end{array}$ \\
\hline & & & & & & & AS & $\begin{array}{c}1.08 \\
(0.39-2.97) \\
\end{array}$ & $\begin{array}{c}1.07 \\
(0.34-3.46) \\
\end{array}$ & $\begin{array}{c}1.13 \\
(0.28-4.56) \\
\end{array}$ & $\begin{array}{c}1.19 \\
(0.28-5.10) \\
\end{array}$ & \begin{tabular}{|c|}
1.23 \\
$(0.33-4.62)$ \\
\end{tabular} & $\begin{array}{c}2.58 \\
(1.08-6.39) \\
\end{array}$ & $\begin{array}{c}75.59 \\
(4.71-1783.80) \\
\end{array}$ \\
\hline & & & & & & & & BV & $\begin{array}{c}0.99 \\
(0.41-2.40)\end{array}$ & $\begin{array}{c}1.05 \\
(0.31-3.30) \\
\end{array}$ & $\begin{array}{c}1.11 \\
(0.30-3.82) \\
\end{array}$ & \begin{tabular}{|c|}
1.15 \\
$(0.37-3.36)$ \\
\end{tabular} & $\begin{array}{c}2.40 \\
(1.51-3.81) \\
\end{array}$ & $\begin{array}{c}69.88 \\
(4.94-1603.59) \\
\end{array}$ \\
\hline & & & & & & & & & BA & $\begin{array}{c}1.05 \\
(0.28-3.84)\end{array}$ & $\begin{array}{c}1.11 \\
(0.27-4.42)\end{array}$ & $\begin{array}{c}1.15 \\
(0.33-3.87)\end{array}$ & $\begin{array}{c}2.41 \\
(1.17-5.10)\end{array}$ & \begin{tabular}{|c|}
70.32 \\
$(4.87-1605.14)$
\end{tabular} \\
\hline & & & & & & & & & & $\mathrm{AM}$ & $\begin{array}{c}1.06 \\
(0.20-5.30)\end{array}$ & $\begin{array}{c}1.09 \\
(0.25-4.77)\end{array}$ & $\begin{array}{c}2.28 \\
(0.79-6.89) \\
\end{array}$ & $\begin{array}{c}66.27 \\
(4.38-1755.93) \\
\end{array}$ \\
\hline & & & & & & & & & & & BS & $\begin{array}{c}1.03 \\
(0.23-4.79)\end{array}$ & $\begin{array}{c}2.16 \\
(0.70-7.33)\end{array}$ & $\begin{array}{c}64.02 \\
(3.74-1579.28)\end{array}$ \\
\hline & & & & & & & & & & & & BJ & $\begin{array}{c}2.10 \\
(0.80-5.68)\end{array}$ & $\begin{array}{c}62.27 \\
(3.82-1469.29)\end{array}$ \\
\hline & & & & & & & & & & & & & $\mathrm{Al}$ & $\begin{array}{c}29.02 \\
(2.19-637.70) \\
\end{array}$ \\
\hline & & & & & & & & & & & & & & $\mathrm{AH}$ \\
\hline
\end{tabular}

Hematological toxicity: $\square$ Regimen $\square$ (95\% Crl)

Figure 5 Selected results of network meta-analysis of grade 3-4 hematological toxicity.

Notes: The treatment regimens are listed in order of grade 3-4 hematological toxicity ranked according to SUCRA. The figure lists only the top 13 chemotherapy regimens (by SUCRA), and it also includes gemcitabine and the least useful regimen as references. Comparisons should be read from left to right. The intersection of the columndefining chemotherapy regimen and the row-defining regimen shows the ORs of grade 3-4 hematological toxicity. For hematological toxicity, an OR value below I favors the row-defining treatment. Reciprocals of corresponding ORs should be taken to get ORs for comparison in the opposing direction. For an explanation of the two-letter codes, please refer to Table 2 .

Abbreviations: $\mathrm{Crl}$, credible interval; OR, odds ratio; SUCRA, surface under the cumulative ranking curve.

and gemcitabine + masitinib (OR 2.41), as shown in Figure 5 and Supplementary S9. Gastrazole (OR 0.03) had the lowest ranked grade 3-4 hematological toxicity compared with gemcitabine.

\section{Ranking}

Supplementary S10 summarizes the ranks of the various regimens for each outcome. For overall survival, NSC-631570 and gemcitabine+NSC-631570 ranked the first and second most efficacious chemotherapy regimens, respectively, with placebo ranking the last. For progression-free survival, PEFG and BAY 12-9566 were the highest ranked. PEFG and gemcitabine+NSC-631570 were the regimens showing the first- and second-ranked overall response rates. PG and FLEC were the most toxic regimens, ranked 1 and 2 for grade 3-4 hematological toxicity.

\section{Model assessment}

As shown in Table 1, no significant heterogeneity of subject baseline characteristics was detected. For most pairwise comparisons, $\mathrm{I}^{2}$ was $<50 \%$ (Supplementary S11). The Markov chains were convergent for each model. The HR and ORs of the traditional pairwise meta-analysis and the pooled results from the network meta-analysis were similar, demonstrating that there were no major inconsistencies in the direct and indirect comparisons (Supplementary S11). The global inconsistency test showed good consistency, with the mean posterior deviance approximating the number of data points for each outcome (Supplementary S12). The results indicated that these models provided a relatively good fit to the data. A comparison-adjusted funnel plot did not reveal any significant publication bias (Supplementary S13).

Three trials had not been included in any network metaanalysis $^{15-17}$ and are summarized in Supplementary S14.

\section{Full use of the data in tables and figures}

Data in the tables and figures provide a comprehensive summary, comparison, and ranking of efficacy and safety results extracted from RCTs evaluating chemotherapy regimens in the last 15 years. They can serve as a reference or provide clues for future clinical practice or research. For example, by referring to the data provided here, RCTs comparing two or more regimens of interest could be identified and the HRs or ORs of achieving various outcomes could be easily found.

The data should be interpreted with care because they provide only an objective summary of the evidence. A chemotherapy regimen ranked first in overall survival may not be the most commonly used in clinical practice. Differences 
between the data obtained in this analysis and those obtained in clinical practice are an important topic for future study.

\section{Discussion}

\section{Summary of evidence}

The prognosis for pancreatic cancer is poor. ${ }^{1}$ For a long time, gemcitabine monotherapy has been the standard first-line treatment for patients with locally advanced and metastatic pancreatic cancer. ${ }^{18}$ More recently, researchers have tried many new antitumor agents and chemotherapy regimens to improve survival. Our Bayesian analysis compared and ranked all the available chemotherapy regimens for locally advanced and metastatic pancreatic adenocarcinoma assessed by RCTs over the last 15 years.

\section{Key results and interpretations}

Our meta-analysis found seven chemotherapy regimens that may achieve better overall survival than gemcitabine monotherapy, with NSC-631570 and gemcitabine+NSC-631570 having the highest rank (HR <0.5). NSC-631570 (Ukrain) is a semi-synthetic compound of the alkaloid chelidonine and thiophosphoric acid. ${ }^{19}$ The use of NSC-631570 and gemcitabine+NSC-631570 as clinical trial chemotherapy regimens for locally advanced and metastatic pancreatic cancer was reported by Gansauge et al in 2002. ${ }^{19}$ This trial included only 90 participants (30 participants per group); nevertheless, both NSC-631570 and gemcitabine+NSC-631570 resulted in better overall survival than gemcitabine, with median overall survival intervals of 5.2 months in the gemcitabine group, 7.9 months in the NSC-631570 group, and 10.4 months in the gemcitabine+NSC-631570 group. However, the following factors limited the clinic application of these two chemotherapy regimens. Side effects such as tumor bleeding could occur after treatment with NSC-631570, and cancer treatment using NSC-631570 should be performed with special medical care. Additionally, the relatively small number of participants means that a large-scale RCT is needed to confirm the superiority and safety of these regimens over gemcitabine monotherapy before they could be used in clinical treatment widely.

PEFG, FLEC, GemCape, gemcitabine + erlotinib, and GS also had a high ranking and achieved improved overall survival compared with gemcitabine, with the longest reported median survival time being $9.3,{ }^{20} 7.9,{ }^{21} 7.1-10.3,{ }^{22-26}$ $6.24-7.6,{ }^{27,28}$ and 8.6-13.7 months, ${ }^{29-33}$ respectively. In comparison, placebo was found to be the least effective regimen in terms of overall survival (HR 7.14), with the median survival time reported as about 4.5 months. ${ }^{34}$ This confirmed the necessity of chemotherapy for locally advanced and metastatic pancreatic cancer.

In our meta-analysis, seven chemotherapy regimens had a high ranking and could achieve improved progression-free survival compared with gemcitabine, with PEFG being the most outstanding (HR 0.51). The trial comparing PEFG and gemcitabine for locally advanced and metastatic pancreatic cancer was reported by Reni et al in 2005. ${ }^{20}$ Their RCT included 99 patients (PEFG, 52 patients; gemcitabine, 47 patients), with PEFG showing a significantly longer median progression-free survival time than gemcitabine (5.4 vs 3.3 months). Care should be taken when interpreting the progression-free survival rankings because only 38 of the 68 studies reported progression-free survival as an outcome. For example, NSC-631570 and gemcitabine+NSC-631570, which had the highest overall survival ranking, were not included in the network meta-analysis of progression-free survival.

\section{Secondary results and interpretations}

Among the seven chemotherapy regimens that had a high ranking and showed an improved overall response rate compared to gemcitabine, PEFG and gemcitabine+NSC-631570 had the highest rank (OR $>7)$. In the study reported by Reni et al, ${ }^{20}$ the overall response rate of PEFG was $38.5 \%$, compared with $8.5 \%$ for gemcitabine. In the study by Gansauge et al, the overall response rate of gemcitabine+NSC-631570 was $21.4 \%$, compared with $3.6 \%$ for gemcitabine. ${ }^{19}$ NSC631570 ranked first and achieved the best disease control rate (OR 7.10), reaching 75.0\%; in the same trial, the disease control rate of gemcitabine was 32.1\%. ${ }^{19}$

Our analysis showed that PG and FLEC ranked the most grade 3-4 hematologically toxic (OR $>4$ ). In a comparison of PG with gemcitabine, grade 3 or 4 neutropenia was significantly more common ( $45.1 \%$ vs $12.8 \%$ ), as were anemia (13.9\% vs $2.9 \%$ ) and thrombocytopenia (17.9\% vs $6.2 \%) .{ }^{35}$ A study comparing FLEC and gemcitabine found grade 3-4 toxicities in the FLEC group of thrombocytopenia (25.3\%), leukopenia (19.7\%), and anemia (14\%), compared with leukopenia $(7.5 \%)$, anemia $(2.9 \%)$, and thrombocytopenia $(1.4 \%)$ in the gemcitabine group. ${ }^{21}$

\section{Other regimens}

This analysis included only regimens in RCTs that included participants with locally advanced and metastatic pancreatic cancer. None of the RCTs included only participants with 
metastatic pancreatic cancer. Several chemotherapy regimens have shown great potential in recent years in the treatment of metastatic pancreatic cancer, especially FOLFIRINOX (oxaliplatin + irinotecan + fluorouracil + leucovorin) and GP (gemcitabine + nab-paclitaxel). ${ }^{36-38}$ In the trial reported by Conroy et al, ${ }^{36}$ the use of FOLFIRINOX resulted in a median overall survival of about 11.1 months among metastatic pancreatic cancer patients (HR $0.57,0.45-0.73$ vs gemcitabine). GP was also found to result in significantly longer median overall survival than gemcitabine in metastatic pancreatic cancer patients (8.7 vs 6.6 months, HR $0.72,0.62-0.83$ ). ${ }^{37,38}$ These trials only included metastatic patients. The 5-year overall survival rates for locally advanced and metastatic pancreatic cancer were $11 \%$ and $3 \%$, respectively, ${ }^{1}$ with significantly different prognoses. Nevertheless, these trials provide some indications for future study, but large-scale RCTs are needed to demonstrate the same results among all locally advanced and metastatic pancreatic cancer patients. To address the problem that some commonly used chemotherapy regimens were not included in the analysis, a similar Bayesian analysis of systemic chemotherapy for metastatic pancreatic cancer was performed. We think the combined used of these two studies would provide relatively comprehensive information for advanced pancreatic cancer.

\section{Strengths}

The present study had several strengths. First, using a network meta-analysis allowed us to obtain indirect comparisons that traditional pairwise could not achieve, as well as more precise effect estimates through combining the direct and indirect comparisons. ${ }^{2-4}$ Second, the study assessed all available RCTs and chemotherapy regimens individually and together, instead of grouping these chemotherapy regimens into several groups. Third, the study assessed the chemotherapy regimens in regard to a range of important outcomes, providing new insights into the benefit-risk ratios of the various treatments. Fourth, we combined studies with different measures into a single model, avoiding potential publication bias..$^{5}$ This updated comprehensive summary of the existing evidence provides clues and reference data for use in future research and clinical practice.

\section{Limitations}

This study has some limitations. First, it only included RCTs published in the last 15 years; the reasons for this are listed in Supplementary S1. Second, there was a certain degree of heterogeneity. An attempt was made to control this heterogeneity. A random-effects model was used for the network analysis of primary outcomes in the main text. The results of a fixed-effects model are shown in Supplementary S6-S9. Third, there may be differences in the results of the network meta-analysis and those obtained in clinical practice. This is discussed in the last paragraph of the "Results" section. Some commonly used chemotherapy clinical regimens were not found in this meta-analysis. To address this limitation, a Bayesian meta-analysis of metastatic pancreatic adenocarcinoma is planned. Fourth, we decided not to perform a meta-analysis for non-hematological toxicity (the reasons are given in Supplementary S2). Fifth, we did not undertake subgroup meta-analyses or meta-regressions because most of the direct one-to-one comparisons were included only in single studies. Sixth, we were unable to construct a contribution matrix and test local inconsistency because too many direct and indirect comparisons were involved. For example, the meta-analysis of overall survival included 54 regimens, which would result in 70 direct comparisons and 1,361 indirect comparisons, leading to 100,170 variables. Most comparisons were conducted in only one study and few loops were found in the network meta-analysis; these factors make the contribution matrix and test of local inconsistency less important. Finally, our meta-analysis used published data rather than individual patient data. Despite these limitations, the comprehensive results of the study, when interpreted with some caution, can provide insights regarding the chemotherapy regimens for locally advanced and metastatic pancreatic cancer, both for future research and for clinical practice.

\section{Conclusion}

Our network meta-analysis found that, of the 63 regimens considered, 7 chemotherapy regimens that may achieve better overall survival than gemcitabine monotherapy for locally advanced and metastatic pancreatic cancer patients, with NSC-631570 and gemcitabine+NSC-631570 having the highest rank. Also, seven chemotherapy regimens had a high ranking and could achieve improved progression-free survival compared with gemcitabine, with PEFG being the most outstanding. PEFG and gemcitabine+NSC-631570 ranked first in overall response and NSC-631570 ranked first in disease control. PG and FLEC had the highest grade 3-4 hematological toxicity. The results should be interpreted and applied with care; and the effectiveness and safety of some chemotherapy regimens need to be further confirmed by large-scale RCTs. 


\section{Acknowledgments}

The project was funded by CAMS Initiative Fund for Medical Sciences (CIFMS) (no. 2016-I2M-1-001). We thank all patients who took part in the trials and all researchers who contributed to the trials. The meta-analysis would not have been possible without patients' and researchers' contribution.

\section{Disclosure}

The authors report no conflicts of interest in this work.

\section{References}

1. Colucci G, Giuliani F, Gebbia V, et al. Gemcitabine alone or with cisplatin for the treatment of patients with locally advanced and/or metastatic pancreatic carcinoma: a prospective, randomized phase III study of the Gruppo Oncologia dell'Italia Meridionale. Cancer. 2002;94(4):902-910.

2. Bramhall SR, Schulz J, Nemunaitis J, Brown PD, Baillet M, Buckels JA. A double-blind placebo-controlled, randomised study comparing gemcitabine and marimastat with gemcitabine and placebo as first line therapy in patients with advanced pancreatic cancer. British journal of cancer. 2002;87(2):161-167.

3. Maisey N, Chau I, Cunningham D, et al. Multicenter randomized phase III trial comparing protracted venous infusion (PVI) fluorouracil (5-FU) with PVI 5-FU plus mitomycin in inoperable pancreatic cancer. Journal of clinical oncology : official journal of the American Society of Clinical Oncology. 2002;20(14):3130-3136.

4. Berlin JD, Catalano P, Thomas JP, Kugler JW, Haller DG, Benson AB, 3rd. Phase III study of gemcitabine in combination with fluorouracil versus gemcitabine alone in patients with advanced pancreatic carcinoma: Eastern Cooperative Oncology Group Trial E2297. Journal of clinical oncology : official journal of the American Society of Clinical Oncology. 2002;20(15):3270-3275.

5. Ducreux M, Rougier P, Pignon JP, et al. A randomised trial comparing 5-FU with 5-FU plus cisplatin in advanced pancreatic carcinoma. Annals of oncology : official journal of the European Society for Medical Oncology. 2002;13(8):1185-1191.

6. Smith D, Gallagher N. A phase II/III study comparing intravenous ZD9331 with gemcitabine in patients with pancreatic cancer. European journal of cancer (Oxford, England : 1990). 2003;39(10):1377-1383.

7. Moore MJ, Hamm J, Dancey J, et al. Comparison of gemcitabine versus the matrix metalloproteinase inhibitor BAY 12-9566 in patients with advanced or metastatic adenocarcinoma of the pancreas: a phase III trial of the National Cancer Institute of Canada Clinical Trials Group. Journal of clinical oncology : official journal of the American Society of Clinical Oncology. 2003;21(17):3296-3302.

8. Ducreux M, Mitry E, Ould-Kaci M, et al. Randomized phase II study evaluating oxaliplatin alone, oxaliplatin combined with infusional 5-FU, and infusional 5-FU alone in advanced pancreatic carcinoma patients. Annals of oncology : official journal of the European Society for Medical Oncology. 2004;15(3):467-473.

9. Cutsem E, Velde H, Karasek P, et al. Phase III trial of gemcitabine plus tipifarnib compared with gemcitabine plus placebo in advanced pancreatic cancer. Journal of clinical oncology : official journal of the American Society of Clinical Oncology. 2004;22(8):1430-1438. http://onlinelibrary.wiley.com/o/cochrane/clcentral/articles/333/ CN-00468333/frame.html.

10. Rocha Lima CM, Green MR, Rotche R, et al. Irinotecan plus gemcitabine results in no survival advantage compared with gemcitabine monotherapy in patients with locally advanced or metastatic pancreatic cancer despite increased tumor response rate. Journal of clinical oncology : official journal of the American Society of Clinical Oncology. 2004;22(18):3776-3783.
11. Cantore M, Fiorentini G, Luppi G, et al. Gemcitabine versus FLEC regimen given intra-arterially to patients with unresectable pancreatic cancer: a prospective, randomized phase III trial of the Italian Society for Integrated Locoregional Therapy in Oncology. Journal of chemotherapy (Florence, Italy). 2004;16(6):589-594.

12. Chen J, Rocken C, Nitsche B, et al. The tyrosine kinase inhibitor imatinib fails to inhibit pancreatic cancer progression. Cancer letters. 2006;233(2):328-337.

13. Louvet C, Labianca R, Hammel P, et al. Gemcitabine in combination with oxaliplatin compared with gemcitabine alone in locally advanced or metastatic pancreatic cancer: results of a GERCOR and GISCAD phase III trial. Journal of clinical oncology : official journal of the American Society of Clinical Oncology. 2005;23(15): 3509-3516.

14. Di Costanzo F, Carlini P, Doni L, et al. Gemcitabine with or without continuous infusion 5-FU in advanced pancreatic cancer: a randomised phase II trial of the Italian oncology group for clinical research (GOIRC). British journal of cancer. 2005;93(2):185-189.

15. Chau I, Cunningham D, Russell C, et al. Gastrazole (JB95008), a novel CCK2/gastrin receptor antagonist, in the treatment of advanced pancreatic cancer: results from two randomised controlled trials. British journal of cancer. 2006;94(8):1107-1115.

16. Richards D, Boehm K, Waterhouse D, et al. Gemcitabine plus CI-994 offers no advantage over gemcitabine alone in the treatment of patients with advanced pancreatic cancer: results of a phase II randomized, double-blind, placebo-controlled, multicenter study. Annals of oncology : official journal of the European Society for Medical Oncology. 2006;17(7):1096-1102. http://onlinelibrary.wiley.com/o/cochrane/ clcentral/articles/464/CN-00570464/frame.html.

17. Stathopoulos GP, Syrigos K, Aravantinos G, et al. A multicenter phase III trial comparing irinotecan-gemcitabine (IG) with gemcitabine (G) monotherapy as first-line treatment in patients with locally advanced or metastatic pancreatic cancer. British journal of cancer. 2006;95(5):587-592.

18. Heinemann V, Quietzsch D, Gieseler F, et al. Randomized phase III trial of gemcitabine plus cisplatin compared with gemcitabine alone in advanced pancreatic cancer. Journal of clinical oncology : official journal of the American Society of Clinical Oncology. 2006;24(24):3946-3952.

19. Abou-Alfa G, Letourneau R, Harker G, et al. Randomized phase III study of exatecan and gemcitabine compared with gemcitabine alone in untreated advanced pancreatic cancer. Journal of clinical oncology : official journal of the American Society of Clinical Oncology. 2006;24(27):4441-4447. http://onlinelibrary.wiley.com/o/cochrane/ clcentral/articles/068/CN-00568068/frame.html.

20. Friess $H$, Langrehr J, Oettle $H$, et al. A randomized multi-center phase II trial of the angiogenesis inhibitor Cilengitide (EMD 121974) and gemcitabine compared with gemcitabine alone in advanced unresectable pancreatic cancer. BMC cancer. 2006;6:285. http://onlinelibrary.wiley. com/o/cochrane/clcentral/articles/309/CN-00574309/frame.html.

21. Boeck S, Hoehler T, Seipelt G, et al. Capecitabine plus oxaliplatin (CapOx) versus capecitabine plus gemcitabine (CapGem) versus gemcitabine plus oxaliplatin (mGemOx): final results of a multicenter randomized phase II trial in advanced pancreatic cancer. Annals of oncology : official journal of the European Society for Medical Oncology. 2008;19(2):340-347.

22. Cascinu S, Berardi R, Labianca R, et al. Cetuximab plus gemcitabine and cisplatin compared with gemcitabine and cisplatin alone in patients with advanced pancreatic cancer: a randomised, multicentre, phase II trial. The Lancet Oncology. 2008;9(1):39-44.

23. Wiedenmann B, Malfertheiner P, Friess H, et al. A multicenter, phase II study of infliximab plus gemcitabine in pancreatic cancer cachexia. The journal of supportive oncology. 2008;6(1):18-25.

24. Spano JP, Chodkiewicz C, Maurel J, et al. Efficacy of gemcitabine plus axitinib compared with gemcitabine alone in patients with advanced pancreatic cancer: an open-label randomised phase II study. Lancet (London, England). 2008;371(9630):2101-2108. 
25. Eckhardt S, Porre P, Smith D, et al. Patient-reported outcomes as a component of the primary endpoint in a double-blind, placebo-controlled trial in advanced pancreatic cancer. Journal of pain and symptom management. 2009;37(2):135-143. http://onlinelibrary.wiley.com/o/ cochrane/clcentral/articles/229/CN-00682229/frame.html.

26. Poplin E, Feng Y, Berlin J, et al. Phase III, randomized study of gemcitabine and oxaliplatin versus gemcitabine (fixed-dose rate infusion) compared with gemcitabine (30-minute infusion) in patients with pancreatic carcinoma E6201: a trial of the Eastern Cooperative Oncology Group. Journal of clinical oncology : official journal of the American Society of Clinical Oncology. 2009;27(23):3778-3785.

27. Saif MW, Oettle H, Vervenne WL, et al. Randomized double-blind phase II trial comparing gemcitabine plus LY293111 versus gemcitabine plus placebo in advanced adenocarcinoma of the pancreas. Cancer journal (Sudbury, Mass). 2009;15(4):339-343.

28. Richards DA, Kuefler PR, Becerra C, et al. Gemcitabine plus enzastaurin or single-agent gemcitabine in locally advanced or metastatic pancreatic cancer: results of a phase II, randomized, noncomparative study. Investigational new drugs. 2011;29(1):144-153.

29. Cunningham D, Chau I, Stocken DD, et al. Phase III randomized comparison of gemcitabine versus gemcitabine plus capecitabine in patients with advanced pancreatic cancer. Journal of clinical oncology : official journal of the American Society of Clinical Oncology. 2009;27(33):5513-5518

30. Meyer T, Caplin ME, Palmer DH, et al. A phase Ib/IIa trial to evaluate the CCK2 receptor antagonist Z-360 in combination with gemcitabine in patients with advanced pancreatic cancer. European journal of cancer (Oxford, England : 1990). 2010;46(3):526-533.

31. Colucci G, Labianca R, Di Costanzo F, et al. Randomized phase III trial of gemcitabine plus cisplatin compared with single-agent gemcitabine as first-line treatment of patients with advanced pancreatic cancer: The GIP-1 study. Journal of Clinical Oncology. 2010;28(10):1645-1651.

32. Kindler HL, Niedzwiecki D, Hollis D, et al. Gemcitabine plus bevacizumab compared with gemcitabine plus placebo in patients with advanced pancreatic cancer: phase III trial of the Cancer and Leukemia Group B (CALGB 80303). Journal of clinical oncology : official journal of the American Society of Clinical Oncology. 2010;28(22):3617-3622.

33. Philip PA, Benedetti J, Corless CL, et al. Phase III study comparing gemcitabine plus cetuximab versus gemcitabine in patients with advanced pancreatic adenocarcinoma: Southwest Oncology Group-directed intergroup trial S0205. Journal of clinical oncology : official journal of the American Society of Clinical Oncology. 2010;28(22):3605-3610.

34. Kindler HL, Ioka T, Richel DJ, et al. Axitinib plus gemcitabine versus placebo plus gemcitabine in patients with advanced pancreatic adenocarcinoma: a double-blind randomised phase 3 study. The Lancet Oncology. 2011;12(3):256-262.

35. Reni M, Cereda S, Rognone A, et al. A randomized phase II trial of two different 4-drug combinations in advanced pancreatic adenocarcinoma cisplatin, capecitabine, gemcitabine plus either epirubicin or docetaxel (PEXG or PDXG regimen). Cancer chemotherapy and pharmacology. 2012;69(1):115-123.

36. Heinrich JC, Tuukkanen A, Schroeder M, Fahrig T, Fahrig R. RP101 (brivudine) binds to heat shock protein HSP27 (HSPB1) and enhances survival in animals and pancreatic cancer patients. Journal of cancer research and clinical oncology. 2011;137(9):1349-1361.

37. Lohr JM, Haas SL, Bechstein WO, et al. Cationic liposomal paclitaxel plus gemcitabine or gemcitabine alone in patients with advanced pancreatic cancer: a randomized controlled phase II trial. Annals of oncology : official journal of the European Society for Medical Oncology. 2012;23(5):1214-1222.

38. Maraveyas A, Waters J, Roy R, et al. Gemcitabine versus gemcitabine plus dalteparin thromboprophylaxis in pancreatic cancer. European journal of cancer (Oxford, England : 1990). 2012;48(9):1283-1292.
39. Gilliam AD, Broome P, Topuzov EG, et al. An international multicenter randomized controlled trial of G17DT in patients with pancreatic cancer. Pancreas. 2012;41(3):374-379.

40. Ozaka M, Matsumura Y, Ishii H, et al. Randomized phase II study of gemcitabine and S-1 combination versus gemcitabine alone in the treatment of unresectable advanced pancreatic cancer (Japan Clinical Cancer Research Organization PC-01 study). Cancer chemotherapy and pharmacology. 2012;69(5):1197-1204.

41. Goncalves A, Gilabert M, Francois E, et al. BAYPAN study: a doubleblind phase III randomized trial comparing gemcitabine plus sorafenib and gemcitabine plus placebo in patients with advanced pancreatic cancer. Annals of oncology : official journal of the European Society for Medical Oncology. 2012;23(11):2799-2805.

42. Meng Z, Garrett CR, Shen Y, et al. Prospective randomised evaluation of traditional Chinese medicine combined with chemotherapy: a randomised phase II study of wild toad extract plus gemcitabine in patients with advanced pancreatic adenocarcinomas. British journal of cancer. 2012;107(3):411-416.

43. Hong JY, Nam EM, Lee J, et al. Randomized double-blinded, placebocontrolled phase II trial of simvastatin and gemcitabine in advanced pancreatic cancer patients. Cancer chemotherapy and pharmacology. 2014;73(1):125-130.

44. Cascinu S, Berardi R, Sobrero A, et al. Sorafenib does not improve efficacy of chemotherapy in advanced pancreatic cancer: A GISCAD randomized phase II study. Digestive and liver disease : official journal of the Italian Society of Gastroenterology and the Italian Association for the Study of the Liver. 2014;46(2):182-186.

45. Bergmann L, Maute L, Heil G, et al. A prospective randomised phase-II trial with gemcitabine versus gemcitabine plus sunitinib in advanced pancreatic cancer: a study of the CESAR Central European Society for Anticancer Drug Research-EWIV. European journal of cancer (Oxford, England : 1990). 2015;51(1):27-36.

46. Borad MJ, Reddy SG, Bahary N, et al. Randomized Phase II Trial of Gemcitabine Plus TH-302 Versus Gemcitabine in Patients With Advanced Pancreatic Cancer. Journal of clinical oncology : official journal of the American Society of Clinical Oncology. 2015;33(13):1475-1481.

47. Bendell J, O'Reilly EM, Middleton MR, et al. Phase I study of olaparib plus gemcitabine in patients with advanced solid tumours and comparison with gemcitabine alone in patients with locally advanced/metastatic pancreatic cancer. Annals of oncology : official journal of the European Society for Medical Oncology. 2015;26(4):804-811.

48. Deplanque G, Demarchi M, Hebbar M, et al. A randomized, placebocontrolled phase III trial of masitinib plus gemcitabine in the treatment of advanced pancreatic cancer. Annals of oncology : official journal of the European Society for Medical Oncology. 2015;26(6):1194-1200.

49. Yamaue H, Tsunoda T, Tani M, et al. Randomized phase II/III clinical trial of elpamotide for patients with advanced pancreatic cancer: PEGASUS-PC Study. Cancer science. 2015;106(7):883-890.

50. Kordes S, Pollak MN, Zwinderman AH, et al. Metformin in patients with advanced pancreatic cancer: a double-blind, randomised, placebocontrolled phase 2 trial. The Lancet Oncology. 2015;16(7):839-847.

51. Dalgleish AG, Stebbing J, Ja Adamson D, et al. Randomised, open-label, phase II study of gemcitabine with and without IMM-101 for advanced pancreatic cancer. British journal of cancer. 2016;115(7):789-796.

52. Laquente B, Lopez-Martin J, Richards D, et al. A phase II study to evaluate LY2603618 in combination with gemcitabine in pancreatic cancer patients. BMC Cancer. 2017;17(1):137.

53. Middleton G, Palmer DH, Greenhalf W, et al. Vandetanib plus gemcitabine versus placebo plus gemcitabine in locally advanced or metastatic pancreatic carcinoma (ViP): a prospective, randomised, double-blind, multicentre phase 2 trial. The Lancet Oncology. 2017;18(4):486-499. 


\section{Publish your work in this journal}

Cancer Management and Research is an international, peer-reviewed open access journal focusing on cancer research and the optimal use of preventative and integrated treatment interventions to achieve improved outcomes, enhanced survival and quality of life for the cancer patient. The manuscript management system is completely online and includes a very quick and fair peer-review system, which is all easy to use. Visit http://www.dovepress.com/testimonials.php to read real quotes from published authors.

Submit your manuscript here: https://www.dovepress.com/cancer-management-and-research-journal 